\title{
HOMOLOGY OF SPACES WITH OPERATORS. I
}

\author{
BY \\ SAMUEL EILENBERG
}

INTRODUCTION

The object of this paper is to study simultaneous invariants of a topological space $X$ and of an abstract group $W$ acting as a group of transformations on $X$. We assume that $W$ also acts as a group of operators on the coefficient group $G$. In addition to the ordinary homology groups of $X$ over $G$ we define two "special" ones that we call the equivariant and the residual homology groups. The three kinds of homology groups are connected by homomorphisms which form an exact sequence, that is, a sequence in which each homomorphism has as kernel the image of the preceding homomorphism. The same is done with cohomologies.

We use singular homology theory [3](1) throughout; this permits us to drop all finiteness assumptions on $W$. In this respect our approach seems to differ from the one very successfully used by P. A. Smith [16].

In Chapter I we develop the theory of abstract complexes with operators. In Chapter II we define the special groups for a topological space $X$ and show that if $X$ is a simplicial complex and if $W$ operates on $X$ simplicially, then the special groups can be computed from the simplicial scheme of $X$. In the last part of Chapter II we define for each (discrete) group $W$ an abstract complex $K_{W}$ which has $W$ as a group of operators. The equivarient groups of $K_{W}$ are shown to be isomorphic with the homology and cohomology groups of the group $W$ studied by Eilenberg and MacLane $[5,6,7]$ and Hopf [10].

In Chapter III we assume that $W$ operates on $X$ without fixed points and that the ordinary homology groups of $X$ vanish up to a certain dimension. It is then shown that the equivariant and residual groups of the space $X$ are isomorphic with the appropriate groups of the group $W$. In Chapter IV these results are applied to prove some theorems about continuous mappings of spaces with operators.

In Chapter $\mathrm{V}$ we take up the homology theory with local coefficient introduced recently by Steenrod [17]. Roughly speaking a homology (or cohomology) group with local coefficients arises whenever the fundamental group $\pi_{1}(X)$ operates on the coefficient group $G$. If $X \sim$ denotes the universal covering space of $X$, then $W=\pi_{1}(X)$ operates both on $X \sim$ and on $G$ thus giving rise to the equivariant groups of $X \sim$. We show that the equivariant

Presented to the Society, April 29, 1944 under the title Skew-invariant cohomology groups; received by the editors May 20, 1946.

(1) Numbers in brackets refer to the bibliography at the end of the paper. 
homology and cohomology groups of $X \sim$ thus obtained coincide with the appropriate groups of $X$ defined using local coefficients.

Combining this result with the main theorem of Chapter III we prove that if the homology groups $\pi_{i}(X)$ of $X$ vanish for $1<i<q$, then the fundamental group $\pi_{1}(X)$ determines the homology and cohomology groups of $X$ with local coefficients in the dimensions less than $q$, and partially determines the groups also in the dimension $q$. This generalizes to local coefficients a theorem of Eilenberg and MacLane [5, 6]. Partially overlapping theorems have also been found (for simple coefficients) independently by Hopf [10], Freudenthal [8], and Eckmann [1].

The following conventions are used throughout the paper. Homology groups have the dimension written as a subscript and have discrete abelian groups as coefficients. Whenever the coefficient group has operators, the operators are written on the right. Cohomology groups have the dimension written as a superscript and have a topological abelian group as coefficients. Whenever the coefficient group has operators, they are left operators. The homology groups are discrete while the cohomology groups are topologized.

\section{Chapter I. Abstract complexes with operators}

1. Closure finite complexes with operators. Let $K$ be a collection of abstract elements $\sigma_{q}$ called cells. With each cell there is associated an integer $q \geqq 0$ called the dimension of $\sigma_{q}$. To any two cells $\sigma_{q+1}, \sigma_{q}$ there corresponds an integer $\left[\sigma_{q+1}: \sigma_{q}\right]$ called the incidence number. $K$ will be called a closure finite abstract complex provided the incidence numbers satisfy the following conditions:

$$
\begin{gathered}
\text { Given } \sigma_{q+1},\left[\sigma_{q+1}: \sigma_{q}\right] \neq 0 \text { only for a finite number of } q \text {-cells } \sigma_{q} ; \\
\text { Given } \sigma_{q+1} \text { and } \sigma_{q-1}, \sum_{\sigma_{q}}\left[\sigma_{q+1}: \sigma_{q}\right]\left[\sigma_{q}: \sigma_{q-1}\right]=0
\end{gathered}
$$

The $q$-cells $\sigma_{q}$ are taken as free generators of an abelian group $C_{q}(K)$; the elements $c_{q}$ of $C_{q}(K)$ will be called (finite) integral $q$-dimensional chains of $K$. The boundary operator $\partial$ is a homomorphism

$$
\partial: C_{q}(K) \rightarrow C_{q-1}(K),
$$

defined for each generator $\sigma_{q}$ as

$$
\partial \sigma_{q}=\sum\left[\sigma_{q}: \sigma_{q-1}\right] \sigma_{q-1} .
$$

Condition (1.1) insures that the summation is finite and therefore that $\partial \sigma_{q}$ is a well defined $(q-1)$-chain. We verify that condition (1.2) is equivalent with

$$
\partial \partial=0 \text {. }
$$

Most of ten in defining a complex we define the boundary operation (1.3) and prove (1.5). The incidence numbers can then be defined by (1.4). Proposi- 
tions (1.1) and (1.2) can then be proved.

Let $K_{1}$ and $K_{2}$ be two abstract closure finite complexes and let $\tau$ be a collection of homomorphisms, one for each dimension $q$,

$$
\tau: C_{q}\left(K_{1}\right) \rightarrow C_{q}\left(K_{2}\right) \text {. }
$$

We say that $\tau$ is a chain transformation $\tau: K_{1} \rightarrow K_{2}$ if

$$
\tau \partial=\partial \tau \text {. }
$$

More specifically, consider the diagram

$$
\begin{array}{ccc}
C_{q}\left(K_{1}\right) & \rightarrow & C_{q}\left(K_{2}\right) \\
\partial \downarrow & & \downarrow \partial \\
C_{q-1}\left(K_{1}\right) & \rightarrow & C_{q-1}\left(K_{2}\right)
\end{array}
$$

Condition (1.7) means that the two mappings of $C_{q}\left(K_{1}\right)$ into $C_{q-1}\left(K_{2}\right)$ that can be derived from the diagram are equal.

Given chain transformations $\tau_{1}: K_{1} \rightarrow K_{2}$ and $\tau_{2}: K_{2} \rightarrow K_{3}$ the composite transformation $\tau_{2} \tau_{1}: K_{1} \rightarrow K_{\mathbf{3}}$ is readily defined.

Let $W$ be an abstract group written multiplicatively. We shall say that $W$ operates on the complex $K$ (on the left) or that $K$ is a complex with operators $W$, provided for every $w \in W$ a chain transformation $w: K \rightarrow K$ is given such that for every $c_{q} \in C_{q}(K)$

$$
\begin{aligned}
w_{2}\left(w_{1} c_{q}\right) & =\left(w_{2} w_{1}\right) c_{q}, \\
1 c_{q} & =c_{q} .
\end{aligned}
$$

If $w c_{q}=c_{q}$ for all $w \in W$ and all chains $c_{q}$, then we say that $W$ operates simply on $K$.

2. Homology groups. Let $G$ be a discrete abelian (additive) group, and let $K$ be an abstract complex as in $\S 1$. The $q$-dimensional chains of $K$ over $G$ are the formal finite sums

$$
c_{q}=\sum g_{i} \sigma_{q}^{i}, \quad \quad g_{i} \in G .
$$

They form an abelian group $C_{q}(K, G)\left({ }^{2}\right)$. Clearly for $g \in G$ and $c_{q} \in C_{q}(K)$ the chain $g c_{q} \in C_{q}(K, G)$ is defined. The boundary homomorphism

$$
\partial: C_{q}(K, G) \rightarrow C_{q-1}(K, G)
$$

is defined as

$$
\partial c_{q}=\sum g_{i} \partial \sigma_{i}^{q}
$$

Again we have $\partial \partial=0$.

(2) More precisely $C_{q}(K, G)$ is the tensor product $G \circ C_{q}(K)$. The tensor product of $G \circ H$ of two groups $G$ and $H$ is the additive group generated by pairs $g h, g \in G, h \in H$ with the relations $\left(g_{1}+g_{2}\right) h=g_{1} h+g_{2} h$ and $g\left(h_{1}+h_{2}\right)=g h_{1}+g h_{2}$. 
The kernel of the homomorphism $\partial: C_{q}(K, G) \rightarrow C_{q-1}(K, G)$, for $q>0$, is denoted by $Z_{q}(K, G)$; the elements of this group are called cycles. We also define $Z_{0}(K, G)=C_{0}(K, G)$. The image $\partial C_{q+1}(K, G)$ in $C_{q}(K, G)$ is denoted by $B_{q}(K, G)$. The quotient group

$$
H_{q}(K, G)=Z_{q}(K, G) / B_{q}(K, G)
$$

is the qth homology group of $K$ over $G$. The elements of $H_{q}(K, G)$ are called homology classes. Each cycle $c \in Z_{q}(K, G)$ determines a homology class, namely the coset $c+B_{q}(K, G)$.

Assume now that $K$ is a complex with operators $W$ and that the same group $W$ acts as a group of (right) operators on the coefficient group $G$, that is, that for every $w \in W$ and $g \in G$ the element $g w \in G$ is defined and that

$$
\left(g_{1}+g_{2}\right) w=g_{1} w+g_{2} w, \quad\left(g w_{1}\right) w_{2}=g\left(w_{1} w_{2}\right), g 1=g .
$$

Given a $q$-chain $c=\sum g_{i} \sigma_{a}^{4}$ we define

$$
w c=\sum g_{i}\left(w \sigma_{q}^{i}\right)
$$

for all $w \in W$. Clearly $\partial(w c)=w(\partial c)$.

Consider the subgroup $C_{q}^{\tau}(K, G)$ of $C_{q}(K, G)$ generated by chains of the form

$$
g(w c)-(g w) c
$$

where $g \in G, w \in W$ and $c \in C_{q}(K)$. The elements of $C_{q}^{r}(K, G)$ will be called residual chains. Since $w \partial=\partial w$ the boundary of (2.1) is the chain $g(w \partial c)$ $-(g w) \partial c$ which is again residual. The group $Z_{q}^{r}(K, G)=Z_{q}(K, G) \cap C_{q}^{r}(K, G)$ is called the group of residual $q$-cycles. The group $B_{q}^{r}(K, G)=\partial C_{q+1}^{r}(K, G)$ is called the group of residual $q$-boundaries. The quotient group

$$
H_{q}^{r}(K, G)=Z_{q}^{r}(K, G) / B_{q}^{r}(K, G)
$$

is the $q$ th residual homology group of $K$ over $G$ (with $W$ as operators).

Chains $c \in C_{q}(K, G)$ whose boundary $\partial c$ is residual will be called equivariant $q$-cycles; they form a subgroup $Z_{q}^{e}(K, G)$ (by definition $Z_{0}^{e}(K, G)=C_{0}(K, G)$ ). The elements of the group $B_{q}^{e}(K, G)=B_{q}(K, G)+C_{q}^{r}(K, G)$ will be called equivariant $q$-boundaries. We have $B_{q}^{e}(K, G) \subset Z_{q}^{e}(K, G)$ and the quotient group

$$
H_{q}^{e}(K, G)=Z_{q}^{e}(K, G) / B_{q}^{e}(K, G)
$$

is called the $q$ th equivariant homology group of $K$ over $G$ (with $W$ as operators).

The three types of homology groups just defined are connected by homomorphisms

$$
\overleftarrow{\psi} \cdots \overleftarrow{\partial} H_{q}^{e}(K, G) \overleftarrow{\psi} H_{q}(K, G) \overleftarrow{\phi} H_{q}^{r}(K, G) \overleftarrow{\partial} H_{q+1}^{e}(K, G) \leftarrow \cdots
$$


defined as follows. The inclusions $Z_{q}^{r} \subset Z_{q} \subset Z_{q}^{e}$ and $B_{q}^{r} \subset B_{q} \subset B_{q}^{e}$ define the homomorphisms $\phi$ and $\psi$. If $c \in Z_{q+1}^{\boldsymbol{e}}$ then $\partial c \in Z_{q}^{r}$ and if $c \in B_{q+1}^{\boldsymbol{e}}=B_{q+1}+C_{q+1}^{r}$ then $\partial c \in B_{q}^{r}$ so that the homomorphism $\partial: H_{q+1}^{e} \rightarrow H_{q}^{r}$ is defined.

By a general theorem (see Kelley and Pitcher [13]) the sequence (2.2) is exact, that is, the kernel of each homomorphism is the image of the preceding one and $H_{0}$ is mapped onto $H_{0}^{e}$.

If $W$ operates simply both on $K$ and $G$ then $C_{a}^{r}(K, G)=0$, the residual homology groups all vanish, and the ordinary and equivariant groups coincide.

3. Groups of homomorphisms. Given a discrete group $A$ and a topological abelian group $G$, consider the group $\operatorname{Hom}(A, G)$ consisting of all homomorphisms $f: A \rightarrow G$ with addition defined by $\left(f_{1}+f_{2}\right)(a)=f_{1}(a)+f_{2}(a)$. A topology is introduced in $\operatorname{Hom}(A, G)$ as follows. Given an element $a \in A$ and an open set $U$ of $G$ containing the zero of $G$ we define the set $(a, U) \subset \operatorname{Hom}(A, G)$ by setting $(a, U)=\{f \mid f(a) \in U\}$. Using the sets $(a, U)$ as a complete set of neighbourhoods of zero, a topology in $\operatorname{Hom}(A, G)$ is obtained.

Given a homomorphism $\phi: A \rightarrow B$ mapping $A$ into another discrete group $B$ we define a homomorphism

$$
\phi^{*}: \operatorname{Hom}(B, G) \rightarrow \operatorname{Hom}(A, G)
$$

by setting for $f \in \operatorname{Hom}(B, G)$

$$
\left(\phi^{*} f\right)(a)=f(\phi a) .
$$

LEMMA 3.1. The homomorphism $\phi^{*}$ is strongly continuous, that is, $\phi^{*}$ is continuous and maps open sets of $\operatorname{Hom}(B, G)$ onto open sets of $\phi^{*}[\operatorname{Hom}(B, G)]$.

Proof. Given $(a, U)$ we have $\phi^{*} f \in(a, U)$ if and only if $\phi^{*} f(a)=f(\phi a) \in U$. This implies that $\phi^{*-1}(a, U)=(\phi a, U)$ and $\phi^{*}$ is continuous. Denote for abbreviation $I=\phi^{*}[\operatorname{Hom}(B, G)]$. For every $(b, U)$ we have

$$
\phi^{*}(b, U)=\bigcup_{\phi(a)=b}[(a, U) \cap I]
$$

this proves that $\phi^{*}(b, U)$ is open in $I$ and therefore $\phi^{*}$ is strongly continuous.

CoRollary 3.2. Denote by $K$ the kernel of $\phi^{*}$, then the isomorphism

$$
\phi^{*}: \operatorname{Hom}(B, G) / K \approx \phi^{*}[\operatorname{Hom}(B, G)]
$$

and its inverse are continuous.

4. Cohomology groups. Let $G$ be a topological abelian group (additive) and let $K$ be an abstract complex as in $\$ 1$. The group of $q$-dimensional cochains of $K$ over $G$ is defined by

$$
C^{q}(K, G)=\operatorname{Hom}\left(C_{q}(K), G\right) .
$$

Clearly a cochain $f$ is uniquely defined by its values $f\left(\sigma_{q}\right)$ for the $q$-cells $\sigma_{q}$. 
The coboundary homomorphism

$$
\delta: C^{q}(K, G) \rightarrow C^{q+1}(K, G)
$$

is defined by setting, for $f \in C^{q}(K, G)$ and $c \in C_{q+1}(K)$,

$$
(\delta f)(c)=f(\partial c) \text {. }
$$

Clearly $\delta=\partial^{*}$ in the sense of $\S 3$ so that $\delta$ is strongly continuous. $\partial \partial=0$ implies $\delta \delta=0$.

The $q$-cochains $f$ such that $\delta f=0$ are called $q$-cocycles; they form a group $Z^{q}(K, G)$. The $q$-cochains $f$ such that $f=\delta f^{\prime}$ are called $q$-coboundaries; they form the group $B^{q}(K, G)$ (by definition $B^{0}(K, G)=0$ ). The quotient group

$$
H^{q}(K, G)=Z^{q}(K, G) / B^{q}(K, G)
$$

is called the $q$ th cohomology group of $K$ over $G$.

Assume now that $K$ is a complex with operators $W$ and that the same group $W$ acts also as a group of (left) operators on the group $G$, that is, that for every $g \in G$ and $w \in W$ the element $w g \in G$ is defined and is continuous in $g$ and that $w\left(g_{1}+g_{2}\right)=w g_{1}+w g_{2}, w_{2}\left(w_{1} g\right)=\left(w_{2} w_{1}\right) g, 1 g=g$.

The cochains $f \in C^{q}(K, G)$ which satisfy

$$
f(w c)=w f(c)
$$

for all $w \in W$ and all $c \in C_{q}(K)$ will be called equivariant; they form a subgroup $C_{e}^{q}(K, G)$ of $C^{q}(K, G)$. If $f$ is equivariant then

$$
\delta f\left(w c_{q+1}\right)=f\left(\partial w c_{q+1}\right)=f\left(w \partial c_{q+1}\right)=w\left[f\left(\partial c_{q+1}\right)\right]=w\left[\delta f\left(c_{q+1}\right)\right],
$$

which shows that $\delta f$ is also equivariant. The group $Z_{e}^{q}(K, G)=Z^{q}(K, G)$ $\cap C_{e}^{q}(K, G)$ is called the group of equivariant $q$-cocycles. The group $B_{e}^{q}(K, G)$ $=\delta C_{e}^{q-1}(K, G)$ is called the group of equivariant coboundaries (by definition $\left.B_{e}^{0}(K, G)=0\right)$. The quotient group

$$
H_{e}^{q}(K, G)=Z_{e}^{q}(K, G) / B_{e}^{q}(K, G)
$$

is the equivariant qth cohomology group of $K$ over $G$.

Cochains $f \in C^{q}(K, G)$ with an equivariant coboundary will be called residual cocycles; they form a group $Z_{r}^{q}(K, G)$. The elements of the group $B_{r}^{q}(K, G)=B^{q}(K, G)+C_{e}^{q}(K, G)$ will be called residual coboundaries. The factor group

$$
H_{r}^{q}(K, G)=Z_{r}^{q}(K, \dot{G}) / B_{r}^{q}(K, G)
$$

is called the $q$ th residual cohomology group of $K$ over $G$.

As in $\$ 2$ we have (strongly continuous) homomorphisms

$$
\cdots \underset{\delta}{\rightarrow} H_{e}^{q}(K, \dot{G}) \underset{\phi}{\rightarrow} H^{q}(K, G) \underset{\psi}{\rightarrow} H_{r}^{q}(K, G) \underset{\delta}{\rightarrow} H_{e}^{q+1}(K, G) \rightarrow \cdots
$$


with $\phi$ and $\psi$ defined by the inclusions $Z_{e}^{q} \subset Z^{a} \subset Z_{r}^{q}$ and $B_{e}^{q} \subset B^{q} \subset B_{r}^{q}$ and the third homomorphism given by the coboundary. Again the sequence (4.2) is exact, meaning that the image of every homomorphism coincides with the kernel of the next one and that the $H_{0}^{0}$ is mapped isomorphically into a subgroup of $H^{0}$.

5. Chain transformations and homotopies. We return to the concept of a chain transformation as defined in $\$ 1$. Let $W$ be a group of operators for two abstract complexes $K_{1}$ and $K_{2}$. A chain transformation $\tau: K_{1} \rightarrow K_{2}$ will be called equivariant provided $\tau(w c)=w(\tau c)$ for every integral chain $c$ in $K_{1}$.

Let $G$ be any discrete abelian group with $W$ as right operators. Given any chain $c \in C_{q}\left(K_{1}, G\right)$ of the form $c=\sum g_{i} \sigma_{q}^{4}$, define $\tau c=\sum g_{i} \tau \sigma_{q}^{t}$. Clearly $\tau \partial c=\partial \tau c$ and $\tau w c=w \tau c$, provided $\tau$ is equivariant. This implies that if $\tau$ is equivariant all the ordinary, residual, and equivariant groups of cycles and boundaries in $K_{1}$ over $G$ are mapped into those of $K_{2}$. Consequently homomorphisms

$$
\begin{aligned}
\tau_{q}: H_{q}\left(K_{1}, G\right) \rightarrow & H_{q}\left(K_{2}, G\right), \quad \tau_{q}^{r}: H_{q}^{r}\left(K_{1}, G\right) \rightarrow H_{q}^{r}\left(K_{2}, G\right), \\
& \tau_{q}^{e}: H_{q}^{e}\left(K_{1}, G\right) \rightarrow H_{q}^{e}\left(K_{2}, G\right)
\end{aligned}
$$

are obtained. These homomorphisms together with the previously considered homomorphisms $\phi, \psi$ and $\partial$ give the following diagram

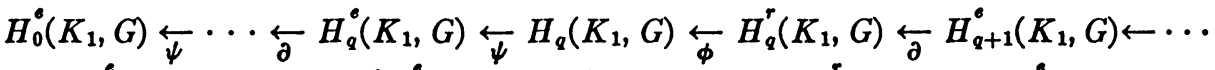

$$
\begin{aligned}
& \downarrow \tau_{0}^{e} \quad \downarrow \tau_{q}^{e} \quad \downarrow \tau_{q} \quad \downarrow \tau_{q}^{r} \quad \downarrow \tau_{q+1}^{\bullet} \\
& H_{0}^{e}\left(K_{2}, G\right) \overleftarrow{\psi} \cdots \overleftarrow{\partial} H_{q}^{e}\left(K_{2}, G\right) \overleftarrow{\psi} H_{q}\left(K_{2}, G\right) \underset{\phi}{H_{q}^{r}}\left(K_{2}, G\right) \overleftarrow{\partial} H_{q+1}^{e}\left(K_{2}, G\right) \leftarrow \cdots
\end{aligned}
$$

It can be shown easily that in the respective squares of the diagram we have commutativity relations

$$
\tau_{q}^{\bullet} \psi=\psi \tau_{q}, \quad \tau_{q} \phi=\phi \tau_{q}^{r}, \quad \tau_{q}^{r} \partial=\partial \tau_{q+1}^{\bullet} .
$$

Passing to cohomologies, let $G$ be a topological abelian group with $W$ as a group of left operators, and let $\tau: K_{1} \rightarrow K_{2}$ be an equivariant chain transformation. Given a cochain $f \in C^{q}\left(K_{2}, G\right)$, we define a cochain $\tau^{*} f \in C^{q}\left(K_{1}, G\right)$ by setting, for all $c \in C_{q}\left(K_{1}\right)$,

$$
\left(\tau^{*} f\right)(c)=f(\tau c) .
$$

This definition of $\tau^{*}$ agrees with the one given in $\S 3$ and therefore the homomorphism $\tau^{*}: C^{q}\left(K_{2}, G\right) \rightarrow C^{q}\left(K_{1}, G\right)$ is strongly continuous. If $f$ is equivariant then

$$
\tau^{*} f(w c)=f(\tau w c)=f(w \tau c)=w f(\tau c)=w\left[\tau^{*} f(c)\right]
$$

and $\tau^{*} f$ also is equivariant. This implies that $\tau^{*}$ generates homomorphisms of all the groups of ordinary equivariant and residual cocycles and coboundaries of 
$K_{2}$ over $G$ into those of $K_{1}$ over $G$. Consequently (strongly continuous) homomorphisms

$$
\begin{aligned}
& \tau^{q}: H^{q}\left(K_{2}, G\right) \rightarrow H^{q}\left(K_{1}, G\right), \quad \tau_{e}^{q}: H_{e}^{q}\left(K_{2}, G\right) \rightarrow H_{e}^{q}\left(K_{1}, G\right), \\
& \tau_{r}^{q}: H_{r}^{q}\left(K_{2}, G\right) \rightarrow H_{r}^{q}\left(K_{1}, G\right)
\end{aligned}
$$

are obtained. Again we have the diagram

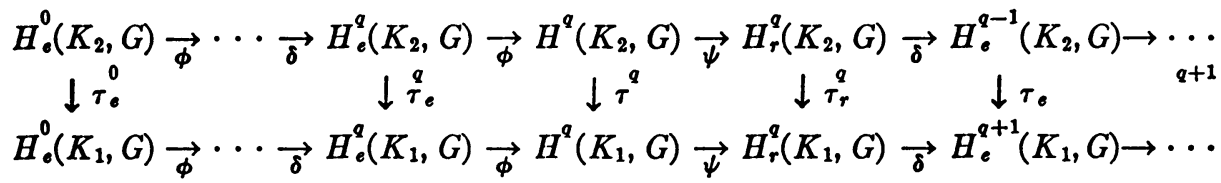

with the commutativity relations

$$
\phi \tau_{e}^{q}=\tau^{q} \phi, \quad \psi \tau^{q}=\tau_{r}^{q} \psi, \quad \delta \tau_{r}^{q}=\tau_{e}^{q+1} \delta .
$$

Two chain transformations $\tau: K_{1} \rightarrow K_{2}$ and $\rho: K_{1} \rightarrow K_{2}$ are said to be chain homotopic if for each $q \geqq 0$ a homomorphism

$$
D: C_{q}\left(K_{1}\right) \rightarrow C_{q+1}\left(K_{2}\right)
$$

is given, such that for each $c \in C_{q}\left(K_{1}\right)$

$$
\partial D c=\tau c-\rho c-D \partial c
$$

(if $q=0$ the last term is omitted). The collection of homomorphisms $D$ is called a chain homotopy.

Given any $c \in G_{q}\left(K_{1}, G\right)$ of the form $c=\sum g_{i} \sigma_{i}^{q}$, define $D c=\sum g_{i} D \sigma_{i}^{q}$. This gives homomorphisms

$$
D: C_{q}\left(K_{1}, G\right) \rightarrow C_{q+1}\left(K_{2}, G\right)
$$

for which (7.1) still holds.

Suppose now that $W$ operates on $K_{1}$ and $K_{2}$ and also on $G$ (on the right). Assume further that $\tau, \rho$, and $D$ are equivariant. The latter condition means of course that $D w c=w D c$. It is then clear that under the homomorphism (5.2) the group $C_{a}\left(K_{1}, G\right)$ maps into $C_{q+1}^{r}\left(K_{2}, G\right)$. Condition (5.1) then implies that if $c$ is a $q$-cycle of any of the three types (ordinary, residual, or equivariant) then $\tau c-\rho c$ is a boundary of the same type. It follows that the homomorphisms $\tau$ and $\rho$ act in the same way upon the three different types of homology groups of $K_{1}$ over $G$.

For the cohomology groups we define, for $q>0$,

$$
D^{*}: C^{q}\left(K_{2}, G\right) \rightarrow C^{q-1}\left(K_{1}, G\right)
$$

by setting, for every $f \in C^{q}\left(K_{2}, G\right)$ and every $c \in C_{q-1}\left(K_{1}\right)$,

$$
D^{*} f(c)=f(D c) \text {. }
$$


Condition (5.1) then becomes

$$
\delta D^{*} f=\tau^{*} f-\rho^{*} f-D^{*} \delta f .
$$

If $\tau, \rho$ and $D$ are equivariant then $D^{*} f$ is equivariant if $f$ is, and formula (5.3) implies that if $f$ is a cocycle of any of the three types then $\tau^{*} f-\rho^{*} f$ is a coboundary of the same type. Hence the homomorphisms $\tau^{*}$ and $\rho^{*}$ have the same effect on the various cohomology groups of $K_{2}$ over $G$.

6. Duality between homology and cohomology. The character group Char $G$ or $G^{-}$of a discrete abelian group $G$ is the group of homomorphisms $\operatorname{Hom}(G, \mathfrak{X})$ where $\mathfrak{X}$ is the additive group of real numbers reduced mod 1 . With the topology of $\S 3, G^{-}$is compact. The value of a character $\chi \in G^{\wedge}$ at $g \in G$ will be written as $(g, \chi)$.

If $W$ is a group of right operators for the group $G$ then setting

$$
(g, w \chi)=(g w, \chi)
$$

we define $w \chi$ for $w \in W$ and $\chi \in G^{\wedge}$ and verify that $W$ becomes a group of left operators on the group $G^{\frown}$.

Let $K$ be an abstract complex with operators $W$. We shall consider homologies in $K$ with coefficients in $G$ and cohomologies in $K$ with coefficients in $G^{-}$ with the operators as above.

Given $c \in C_{q}(K, G)$ and $f \in C^{q}\left(K, G^{\wedge}\right)$ we define an element $(c, f) \in \mathfrak{X}$, called the Kronecker index, as follows. If $c=\sum g_{i} \sigma_{a}^{8}$ then

$$
(c, f)=\sum_{i}\left(g_{i}, f\left(\sigma_{q}^{i}\right)\right) .
$$

The following properties of the Kronecker index are useful

$$
\begin{aligned}
& (c, f) \text { is bilinear and continous in } f, \\
& (c, f)=0 \text { for all } c \text { if and onl } y \text { if } f=0, \\
& (c, f)=0 \text { for all } f \text { if and onl } y \text { if } c=0, \\
& (c, \delta f)=(\partial c, f) \text { for } c \in C_{q+1}(K, G) \text { and } f \in C^{q}\left(K, G^{-}\right) .
\end{aligned}
$$

For a fixed $f \in C^{q}\left(K, G^{-}\right)$the symbol $(c, f)$ defines a character of the group $C_{q}(K, G)$. With this convention $C^{q}\left(K, G^{\wedge}\right)$ actually becomes (see [12, chap. 2]) the group of characters of $C_{q}(K, G)$ :

$$
C^{q}\left(K, G^{\wedge}\right)=\text { Char } C_{q}(K, G) .
$$

We shall now prove the following propositions concerning annihilators.

$$
\begin{aligned}
& Z_{q}(K, G)=\operatorname{Annih} B^{q}\left(K, G^{\varkappa}\right), \quad Z^{q}\left(K, G^{-}\right)=\operatorname{Annih} B_{q}(K, G), \\
& Z_{q}^{e}(K, G)=\operatorname{Annih} B_{e}^{q}\left(K, G^{\uparrow}\right), \quad Z_{e}^{q}\left(K, G^{\varkappa}\right)=\operatorname{Annih} B_{q}^{e}(K, G), \\
& Z_{q}^{r}(K, G)=\operatorname{Annih} B_{r}^{q}\left(K, G^{\curlywedge}\right), \quad Z_{r}^{q}\left(K, G^{\frown}\right)=\operatorname{Annih} B_{q}^{r}(K, G) \text {. }
\end{aligned}
$$


$c \in$ Annih $B^{q}\left(K, G^{\wedge}\right)$ means $(c, \delta f)=0$ for all $f \in C^{q-1}\left(K, G^{\wedge}\right)$. By (6.6) this means $(\partial c, f)=0$ for all $f \in C^{q-1}\left(K, G^{-}\right)$and by (6.4) this means $\partial c=0$. This proves the first part of (6.8). To prove the second, take $f \in$ Annih $B_{q}(K, G)$. This means that $(\partial c, f)=0$ for all $c \in C_{q+1}(K, G)$. By (6.6) this is equivalent with $(c, \delta f)=0$ for all $c \in C_{q+1}(K, G)$ which by (6.4) is equivalent with $\delta f=0$.

Before we proceed with the proof of (6.9) and (6.10) we shall prove that the equivariant cochains are the annihilators of the residual chains:

$$
C_{e}^{q}\left(K, G^{\mathcal{C}}\right)=\text { Annih } C_{q}^{r}(K, G), \quad C_{q}^{r}(K, G)=\operatorname{Annih} C_{e}^{q}\left(K, G^{\frown}\right) .
$$

Let $f \in$ Annih $C_{a}^{r}(K, G)$. This means that $((g w) c-g(w c), f)=0$ for all $g \in G, w \in W$, and $c \in C_{q}(K)$. This is equivalent with $((g w) c, f)=(g(w c), f)$. Using definition (6.2), this is equivalent with $(g w, f(c))=(g, f(w c))$, and using definition (6.1), this is equivalent with $(g, w f(c))=(g, f(w c))$. This last equation will hold for all $g \in G$ if and only if $w f(c)=f(w c)$, that is, if and only if $f$ is equivariant. This proves the first part of (6.11). The other half follows from (6.7) and general theorems on annihilators [14].

We now return to the proof of (6.9) and (6.10). Let $c \in$ Annih $B_{e}^{q}\left(K, G^{\wedge}\right)$. By (6.6) this is equivalent with $(\partial c, f)=0$ for all $f \in C_{e}^{q-1}\left(K, G^{\wedge}\right)$. By (6.11) this is true if and only if $\partial c \in C_{q}^{r}(K, G)$, that is, if and only if $c \in Z_{q}^{b}(K, G)$. This proves the first part of (6.9). Take now $f \in$ Annih $B_{q}^{r}(K, G)$. This means that $(\partial c, f)=0$ for all $c \in C_{a+1}^{r}(K, G)$. By (6.6) this is equivalent with $(c, \delta f)=0$ for all $c \in C_{a+1}^{r}(K, G)$. By (6.11) this is true if and only if $\delta f \in C_{e}^{q+1}(K, G)$, that is, if and only if $f \in Z_{r}^{q}(K, G)$. This proves the second part of (6.10).

To prove the second part of (6.9) and the first part of (6.10) write

and similarly

$$
\text { Annih } \begin{aligned}
B_{q}^{e}(K, G) & =\text { Annih }\left[B_{q}(K, G)+C_{q}^{r}(K, G)\right] \\
& =\text { Annih } B_{q}(K, G) \cap \text { Annih } C_{q}^{r}(K, G) \\
& =Z^{q}\left(K, G^{\frown}\right) \cap C_{e}^{q}\left(K, G^{\frown}\right)=Z_{e}^{q}\left(K, G^{\frown}\right)
\end{aligned}
$$

$$
\text { Annih } \begin{aligned}
B_{r}^{q}\left(K, G^{\frown}\right) & =\operatorname{Annih}\left[B^{q}\left(K, G^{\frown}\right)+C_{e}^{q}\left(K, G^{\frown}\right)\right] \\
& =\operatorname{Annih} B^{q}\left(K, G^{\frown}\right) \cap \operatorname{Annih} C_{e}^{q}\left(K, G^{\frown}\right) \\
& =Z_{q}(K, G) \cap C_{q}^{r}(K, G)=Z_{q}^{r}(K, G) .
\end{aligned}
$$

This concludes the proof of (6.8)-(6.10). From these relations and general theorems about annihilators in character groups the following isomorphisms follow for $G^{\wedge}=$ Char $G$ :

$$
\begin{gathered}
H^{q}\left(K, G^{\frown}\right) \approx \operatorname{Char} H_{q}(K, G), \quad H_{e}^{q}\left(K, G^{\uparrow}\right) \approx \operatorname{Char} H_{q}^{e}(K, G), \\
H_{r}^{q}\left(K, G^{\frown}\right) \approx H_{q}^{r}(K, G) .
\end{gathered}
$$


This shows that the groups in the exact sequence

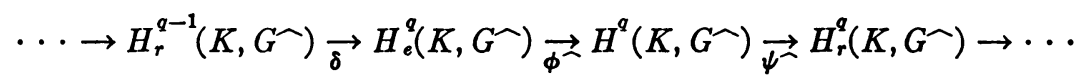

are the character groups of the corresponding groups in the exact sequence

$$
\cdots \leftarrow H_{q-1}^{r}(K, G) \overleftarrow{\partial} H_{q}^{e}(K, G) \overleftarrow{\psi} H_{q}(K, G) \overleftarrow{\phi} H_{q}^{r}(K, G) \leftarrow \cdots
$$

Moreover it could be shown that the homomorphisms in (6.13) and (6.14)

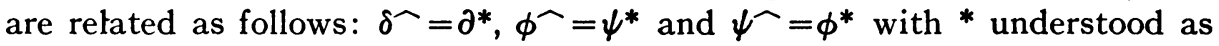
in $\$ 3$.

7. Homologies modulo a subcomplex. An abstract complex $L$ is a subcomplex of an abstract complex $K$ if the cells of $L$ are among the cells of $K$ and if the boundary operator in $L$ agrees with the one in $K$. This implies that $L$ is "closed" in $K$, that is, that if $\left[\sigma_{q}: \sigma_{q-1}\right] \neq 0$ and $\sigma_{q} \in L$ then $\sigma_{q-1} \in L$. A chain of $K$ will be said to be in $L$ (or in $K-L$ ) if it is a linear combination of cells in $L$ (or not in $L$ ).

As usual we define $Z_{q}(K, L, G)$ to be the subgroup of $C_{q}(K, G)$ consisting of the chains $c$ with $\partial c$ in $L$ (by definition $Z_{0}(K, L, G)=C_{0}(K, G)$ ). Also let $B_{q}(K, L, G)=B_{q}(K, G)+C_{q}(L, G)$. The quotient group $H_{q}(K, L, G)$ $=Z_{q}(K, L, G) / B_{q}(K, L, G)$ is the $q$ th homology group of $K$ modulo $L$ over $G$.

As in $\$ 2$ we have an exact sequence (see Kelley and Pitcher [13])

$$
\cdots \leftarrow H_{q}(K, L, G) \leftarrow H_{q}(K, G) \leftarrow H_{q}(L, G) \leftarrow H_{q+1}(K, L, G) \leftarrow \cdots
$$

Suppose now that $W$ is a group operating on the complex $K$. The subcomplex $L$ will be called segregated provided that if $c$ is a chain in $L$ or in $K-L$ then, for any $w \in W, w c$ is also in $L$ or in $K-L$ respectively. If $L$ is segregated then

$$
C_{q}^{r}(L, G)=C_{q}^{r}(K, G) \cap C_{q}(L, G)
$$

for any discrete abelian group $G$ with $W$ as right operators.

We now define the residual and equivariant groups of $K$ modulo $L$. $Z_{a}^{r}(K, L, G)$ is the subgroup of $C_{a}^{r}(K, G)$ of the chains $c$ with $\partial c \in C_{a-1}^{r}(L, G)$ (by definition $\left.Z_{0}^{r}(K, L, G)=C_{0}^{r}(K, G)\right)$ while $B_{q}^{r}(K, L, G)$ is defined to be $B_{q}^{r}(K, G)$ $+C_{q}^{r}(L, G)$. The quotient group $H_{q}^{r}(K, L, G)=Z_{q}^{r}(K, L, G) / B_{q}^{r}(K, L, G)$ is the $q$ th residual homology group of $K$ modulo $L$ over $G$.

The group $Z_{q}^{e}(K, L, G)$ consists of the chains $c \in C_{q}(K, L)$ with $\partial c$ $\in C_{q-1}^{r}(K, G)+C_{q-1}(L, G)$ (by definition $\left.\left(Z_{0}^{e} K, L, G\right)=C_{0}(K, G)\right)$, while $B_{q}^{e}(K, L, G)$ is defined as $B_{q}(K, G)+C_{q}^{r}(K, G)+C_{q}(L, G)$. The equivariant homology groups of $K$ modulo $L$ are defined as quotients $H_{q}^{e}(K, L, G)$ $=Z_{\boldsymbol{q}}^{\boldsymbol{e}}(K, L, G) / B_{\boldsymbol{q}}^{\boldsymbol{e}}(K, L, G)$.

The ordinary, residual and equivariant homology groups of $K, L$ and $K$ 
modulo $L$ (for $L$ segregated) lead to six exact sequences illustrated on the following diagram (the symbol for the coefficient group $G$ is omitted).

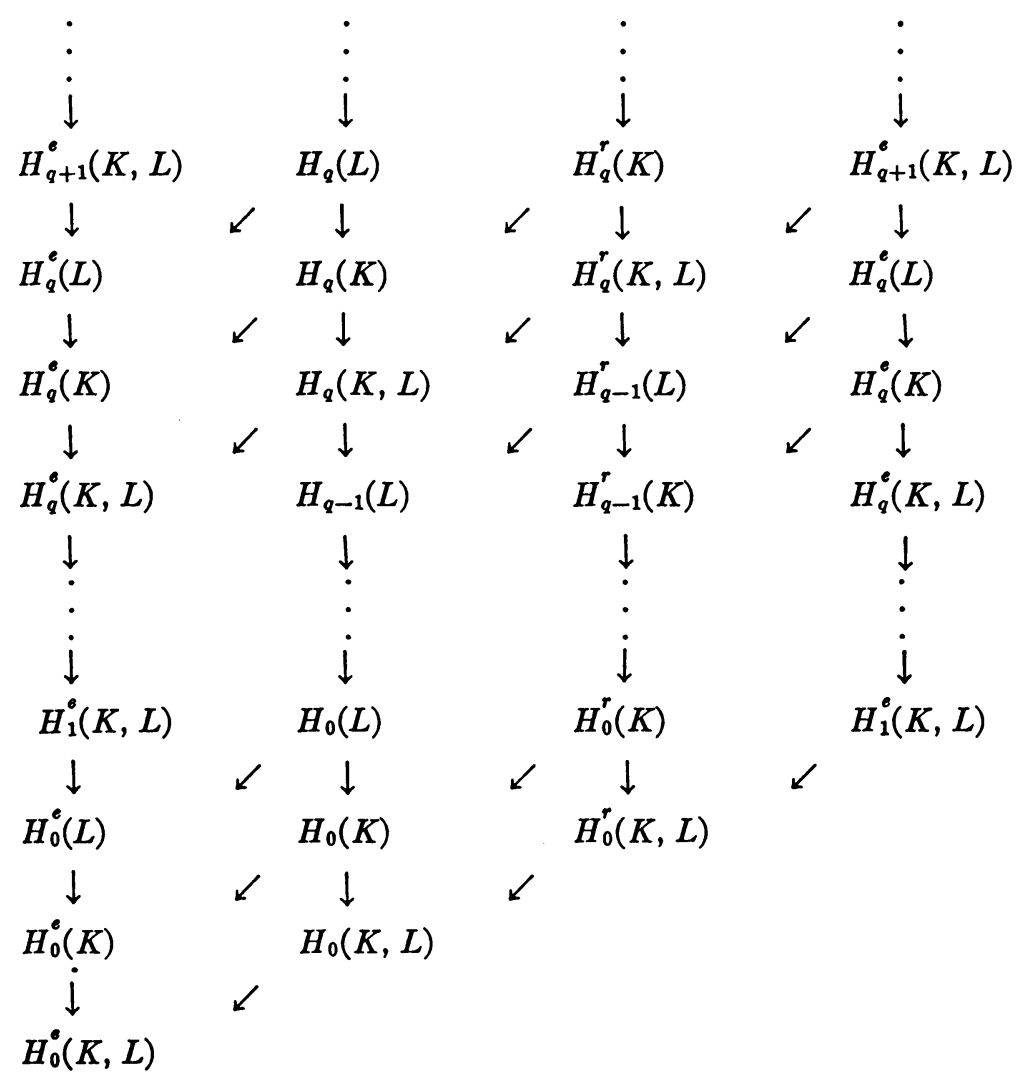

In this diagram the extreme two columns should be identified. In the resulting cylinder one has three exact sequences running vertically and three running spirally. With the homomorphisms explicitly defined it is easy to see that in each parallelogram commutativity relations hold asserting that the two homomorphisms leading from the upper right corner to the lower left corner are equal to each other.

The discussion for cohomologies is quite analogous. The cohomology diagram can be obtained from the preceding one by reversing the direction of all the arrows and interchanging subscripts and superscripts.

Chapter II. Homology TheORY FOR SPACES, POLYHEDRA AND GROUPS

8. The singular complex $S(X)$. A geometric simplex $s$ whose vertices are given a definite order $p_{0}<\ldots<p_{q}$ will be called an ordered simplex and will be written $s=\left\langle p_{0} \cdots p_{q}\right\rangle$. A singular $q$-simplex in a topological space $X$ 
is a continuous mapping $T: s \rightarrow X$ of an ordered $q$-simplex $s$ into $X$. Two singular $q$-simplexes $T_{1}: s_{1} \rightarrow X$ and $T_{2}: s_{2} \rightarrow X$ are equivalent (notation: $T_{1} \equiv T_{2}$ ) provided $T_{2} B=T_{1}$ where $B$ is the barycentric mapping $B: s_{1} \rightarrow s_{2}$ preserving the order. Let $C_{q}(X)$ be the free abelian group generated by the equivalence classes of the $q$-dimensional singular simplexes in $X$. The elements of $C_{q}(X)$ will be called integral $q$-chains in $X$.

We now proceed with the definition of the boundary operator for the chains in $X$. Given $T: s \rightarrow X$, where $s=\left\langle p_{0} \cdots p_{q}\right\rangle(q>0)$, denote by $s^{(i)}$ the $(q-1)$-dimensional face of $s$ which does not contain the vertex $p_{i}$. With the vertices ordered as in $s, s^{(i)}$ is an ordered simplex; the map $T^{(i)}: s^{(i)} \rightarrow X$ obtained by setting $T^{(i)}(x)=T(x)$ for $x \in s^{(i)}$ is then a singular $(q-1)$-simplex in $X$. We define the boundary $\partial T$ to be the chain $\partial T=\sum_{i=0}^{j}(-1)^{i} T^{(i)}$. In writing the above formula we did not distinguish between a singular simplex and its equivalence class. The boundary operator $\partial$ defines a homomorphism $\partial: C_{q}(X) \rightarrow C_{q-1}(X)(q>0)$ and $\partial \partial=0$. Consequently the operation $\partial$ can be used to define a closure finite abstract complex that is denoted by $S(X)$ and called the singular complex of the space $X$. The homology and cohomology groups $H_{q}(X, G)$ and $H^{q}(X, G)$ will by definition be the appropriate homology and cohomology groups of the complex $S(X)$.

If $A$ is a subset of $X$ then $S(A)$ is a subcomplex of $S(X)$ and the homology and cohomology groups of $X$ modulo $A$ are defined to be the appropriate groups of $S(X)$ modulo $S(A)$.

A continuous mapping $\phi: X \rightarrow Y$ of the space $X$ into a space $Y$ induces a chain transformation $\phi: S(X) \rightarrow S(Y)$ defined as follows. For each singular simplex $T: s \rightarrow X$ the singular simplex $\phi T: s \rightarrow Y$ is obtained by the composition of the maps $T$ and $\phi$.

A group $W$ will be called a group of operators for a topological space $X$ provided that for each $w \in W$ and each $x \in X$ an element $w x \in X$ is given which varies continuously with $x$ and such that $1 x=x$ and $\left(w_{1} w_{2}\right) x=w_{1}\left(w_{2} x\right)$. It follows that each $w \in W$ defines a homeomorphism $w: X \rightarrow X$. This homeomorphism in turn defines a chain transformation $w: S(X) \rightarrow S(X)$, so that $W$ acts as a group of operators on the complex $S(X)$. We define the equivariant and residual homology and cohomology groups of $X$ to be the appropriate groups of $S(X)$.

If $A$ is a subset of $X$ such that $w a \in A$ for each $w \in W$ and each $a \in A$, then $A$ is said to be an invariant subspace of $X$. The complex $S(A)$ is then an invariant subcomplex of $S(X)$ and $S(A)$ is segregated in $S(X)$ in the sense of $\$ 7$.

All the definitions and results of the previous chapter, established for complexes, thus are carried over to topological spaces.

9. Simplicial complexes with operators. Let $P$ be a locally finite polyhedron with a fixed simplicial decomposition. If a group $W$ operates on $P$ and if every transformation $w: P \rightarrow P$ is simplicial then we say that $W$ operates 
simplicially on $P$. In addition to the singular complex $S(P)$ we shall define as in [3] two other abstract complexes $k(P)$ and $K(P)$ both of which will be complexes with $W$ as operators.

In order to define $k(P)$ one chooses a definite orientation for every simplex of $P$. The $q$-cells of $k(P)$ are these oriented $q$-simplexes of $P$ and the incidence numbers are defined in the usual way. The simplicial mappings $w: P \rightarrow P$ induce chain transformations $w: k(P) \rightarrow \dot{k}(P)$, and thus $k(P)$ is a complex with operators $W$. Changing the orientation of some or all of the simplexes of $P$ replaces the complex $k(P)$ by another complex $k^{\prime}(P)$. Every cell in $k^{\prime}(P)$ is \pm a cell in $k(P)$. This leads to two equivariant chain transformations

$$
\rho: k(P) \rightarrow k^{\prime}(P), \quad \rho^{\prime}: k^{\prime}(P) \rightarrow k(P)
$$

such that $\rho \rho^{\prime}$ and $\rho^{\prime} \rho$ are identities. This shows that all the various homology and cohomology groups of $k$ and $k^{\prime}$ are isomorphic.

We shall now define the complex $K(P)$ as follows. A $q$-cell of $K(P)$ is an ordered array $v_{0} \cdots v_{q}$ of vertices of $P$ with possible repetitions and with the condition that all the vertices in question have to lie on one geometric simplex of $P$. The boundary is defined to be $\left(^{3}\right)$

$$
\partial\left(v_{0} \cdots v_{q}\right)=\sum_{i=0}^{q}(-1)^{i} v_{0} \cdots v_{i} \cdots v_{q},
$$

since $\partial \partial=0$ the complex $K(P)$ is defined. Setting $w\left(v_{o} \cdots v_{q}\right)=w v_{o} \cdots w v_{q}$ defines a chain transformation $w: K(P) \rightarrow K(P)$ so that $K(P)$ has $W$ as operators.

The three complexes $k(P), K(P)$, and $S(P)$ are compared by means of chain transformations $\alpha: K(P) \rightarrow k(P), \beta: K(P) \rightarrow S(P)$ defined as follows. Let $v_{0} \cdots v_{q}$ be a $q$-cell of $K(P)$. If $v_{0}, \cdots, v_{q}$ are not distinct then $\alpha\left(v_{0} \cdots v_{q}\right)=0$. If the vertices $v_{0}, \cdots, v_{q}$ are distinct then they define uniquely a $q$-cell $\sigma_{q}$ of $k(P)$. Define $\alpha\left(v_{0} \cdots v_{q}\right)= \pm \sigma_{q}$ according as the orientation of $\sigma_{q}$ agrees or disagrees with the order $v_{0}<\cdots<v_{q}$. The singular simplex $\beta\left(v_{0} \cdots v_{q}\right)$ is defined as follows. Let $s=\left\langle p_{0} \cdots p_{q}\right\rangle$ be an ordered $q$-simplex and let $T: s \rightarrow P$ be the barycentric mapping such that $T\left(p_{i}\right)=v_{i}$ for $i=0, \cdots, q$. Then define $\beta\left(v_{0} \cdots v_{q}\right)=T$.

The chain transformations $\alpha$ and $\beta$ possess two important features:

$1^{\circ} . \alpha$ and $\beta$ are equivariant.

$2^{\circ}$. $\alpha$ and $\beta$ are hereditary in the following sense. Let $Q$ be a closed simplicial subpolyhedron of $P$ invariant under $W$. The complexes $k(Q), K(Q)$, and $S(Q)$ are then subcomplexes of $k(P), K(P)$, and $S(P)$ respectively, and the chain transformations $\alpha_{Q}: K(Q) \rightarrow k(Q)$ and $\beta_{Q}: K(Q) \rightarrow S(Q)$ agree with $\alpha$ and $\beta$.

In the sequel we shall discuss conditions under which the chain transfor-

(3) The circumflex to the right of $v_{i}$ indicates that $v_{i}$ is omitted. 
mations $\alpha$ and $\beta$ establish isomorphisms of the various homology and cohomology groups of $k(P), K(P)$, and $S(P)$.

10. Regular operators. The operators $W$ of a simplicial complex $P$ will be called regular provided the fixed points of each transformation $w: P \rightarrow P$ form a subcomplex of $P$. This is equivalent to the assumption that if $w$ maps a simplex $s$ of $P$ on itself then $w x=x$ for each $x \in s$. It is easy to see that $W$ is always regular when regarded as a group of operators on the first barycentric subdivision $P^{\prime}$ of $P$.

THEOREM 10.1. Let $P$ be a simplicial locally finite polyhedron and $W$ a group of regular operators on $P$. There exist equivariant chain transformations

$$
\bar{\alpha}: k(P) \rightarrow K(P), \quad \bar{\beta}: S(P) \rightarrow K(P)
$$

such that

(10.1) $\alpha \bar{\alpha}$ is the identity chain transformation $k(P) \rightarrow k(P)$.

(10.2) $\bar{\alpha} \alpha$ is equivariantly chain homotopic with the identity chain transformation $K(P) \rightarrow K(P)$,

(10.3) $\bar{\beta} \beta$ is the identity chain transformation $K(P) \rightarrow K(P)$,

(10.4) $\beta \bar{\beta}$ is equivariantly chain homotopic with the identity chain transformation $S(P) \rightarrow S(P)$.

Moreover, $\bar{\alpha}$ and $\bar{\beta}$ and the chain homotopies will have the hereditary property described in $\$ 9$.

Theorem 10.1 and the results of $\$ 5$ imply the following:

Corollary 10.2. Let $P$ be a simplicial locally finite polyhedron and $W a$ group of regular operators on $P$. The various homology and cohomology groups of the complexes $k(P), K(P)$, and $S(P)$ are then isomorphic. The isomorphisms are obtained by means of the chain transformations $\alpha: K(P) \rightarrow k(P)$ and $\beta: K(P)$ $\rightarrow S(P)$.

In particular the various groups of $P$ as a space can be computed from the complexes $k(P)$ or $K(P)$. If the operators are not regular the computation can be made using $k\left(P^{\prime}\right)$ or $K\left(P^{\prime}\right)$ where $P^{\prime}$ is the first barycentric subdivision of $P$, or any other subdivision of $P$ on which the operators $W$ are regular. Note that $S(P)=S\left(P^{\prime}\right)$.

An easy example shows that the regularity assumption is not superfluous. Let $P$ be the closed unit interval with the obvious simplicial decomposition (one edge and two vertices). Let $W$ be cyclic of order 2 and let the nontrivial element of $W$ act on $P$ by interchanging the two vertices of $P$. It is then easy to verify that the complexes $k(P)$ and $k\left(P^{\prime}\right)$ (similarly $K(P)$ and $K\left(P^{\prime}\right)$ ) need not have the same equivariant or residual groups. Since the groups of $k\left(P^{\prime}\right)$ (or $K\left(P^{\prime}\right)$ ) are those of $P$ as a space, it follows that the groups of $k(P)$ (or $K(P)$ ) need not be topological invariants of $P$ as a space.

The construction of $\bar{\beta}$ and the proof of (10.3) and (10.4) have been carried 
out elsewhere [4]. We proceed with the construction of $\bar{\alpha}$. This construction varies little from the analogous construction without operators (see $[3, \mathrm{p}$. $419]$ ). Additional care has to be taken to secure the equivariance and the heredity.

By a system of representatives for $P$ we shall understand a collection $\{\bar{s}\}$ of simplexes of $P$ such that any simplex $s$ of $P$ is the $W$-image of exactly one $\bar{s}$. If $w$ and $w^{\prime}$ are two elements of $W$ both of which map $\bar{s}$ into $s$ then $w^{-1} w^{\prime}: \bar{s} \rightarrow \bar{s}$ and since the operators are regular it follows that $w^{\prime}=w$ on $\bar{s}$. This shows that the mapping $\vec{s} \rightarrow s$ is unique even though it may be given by different elements of $W$.

It will be convenient to orient the simplexes of $P$ by first assigning an orientation to the representative simplexes $\bar{s}$ and then orienting the other simplexes using the mappings $\bar{s} \rightarrow s$. Such an orientation will be called concordant. Without any loss of generality we may assume that the complex $k(P)$ is obtained using a concordant orientation.

We define the chain transformation $\bar{\alpha}: k(P) \rightarrow K(P)$ by induction. Since the 0 -cells of $k(P)$ and $K(P)$ coincide we may define $\bar{\alpha}$ to be the identity map on the 0-chains. Suppose that $\bar{\alpha} c$ has been defined for all integral chains $c$ of dimension less than $q$ and has the following properties:

$$
\partial \bar{\alpha} c=\bar{\alpha} \partial c, \quad w \bar{\alpha} c=\bar{\alpha} w c, \quad \alpha \bar{\alpha} c=c .
$$

$\bar{\alpha} \sigma$ is carried by the simplex of $P$ carrying $\sigma$.

Let now $\bar{\sigma}$ be any $q$-cell carried by a representative $q$-simplex $\bar{s}$. Then $\bar{\alpha} \partial \bar{\sigma}$ is defined and is a cycle since $\partial \bar{\alpha} \partial \bar{\sigma}=\bar{\alpha} \partial \partial \bar{\sigma}=0$. Moreover, in view of (10.6), $\bar{\alpha} \partial \bar{\sigma}$ is a cycle in the complex $K(\bar{s})$. Since every cycle of $K(\bar{s})$ bounds we may find a $q$-chain $\bar{\alpha} \bar{\sigma}$ in $K(\bar{s})$ such that $\partial \bar{\alpha} \bar{\sigma}=\bar{\alpha} \partial \bar{\sigma}$. Since $\alpha \bar{\alpha} \bar{\sigma}$ is a chain in $k(\bar{s})$ and $\partial \alpha \bar{\alpha} \bar{\sigma}=\alpha \bar{\alpha} \partial \bar{\sigma}=\partial \bar{\sigma}$ it follows that $\alpha \bar{\alpha} \bar{\sigma}=\bar{\sigma}$. Any nonrepresentative $q$-cell $\sigma$ is the image $w \bar{\sigma}$ of some representative. We define $\bar{\alpha} \sigma=w \bar{\alpha} \bar{\sigma}$. Since $P$ is oriented concordantly this definition is independent of the choice of $w$. This completes the definition of $\bar{\alpha}$.

In order to prove (10.2) we must construct a suitable equivariant chain homotopy $D$. The main fact utilized in the construction is that for every cell $\sigma$ of $K(P)$ the chains $\bar{\alpha} \alpha \sigma$ and $\sigma$ are carried by the same simplex of $P$. The definition of $D$ proceeds by induction and is quite analogous to the definition of $D$ in [3, p. 419]. In order to insure that $D$ is equivariant we select a system of representatives for the cells of $K(P)$, define $D$ first for the representative cells of a given dimension, then extend the definition to other cells by the equivariance condition.

11. Augmentable complexes. Let $K$ be an abstract closure finite complex. Given an integral 0-chain $c=\sum a_{i} \sigma_{0}^{i}$ define $I(c)=\sum a_{i}$. The complex $K$ will be called augmentable if $I(c)=0$ for every boundary $c \in B_{0}(K)$.

The complex $K$ can be augmented by adding a single (-1)-cell $\sigma_{-1}$ with 
the incidence numbers $\left[\sigma_{0}: \sigma_{-1}\right]=1$ for every 0 -cell $\sigma_{0}$. The condition that $K$ is augmentable is then equivalent with the condition that in the augmented complex $\bar{K}$ we have $\partial \partial=0$. Thus we may consider the homology and cohomology groups of $\bar{K}$. For dimension greater than 0 the groups of $K$ and $\bar{K}$ coincide. In the dimension zero the groups will differ. We shall write $\bar{H}_{0}(K, G)$ instead of $H_{0}(\bar{K}, G)\left[\bar{H}^{0}(K, G)\right.$ instead of $\left.H^{0}(\bar{K}, G)\right]$ and call this group the reduced 0th homology [cohomology] group of the complex $K$ over $G$.

The reduced groups $\bar{H}_{0}(K, G)$ and $\bar{H}^{0}(K, G)$ can be defined directly in terms of $K$ as follows. Define $\bar{Z}_{0}(K, G)$ to be the subgroup of the group $Z_{0}(K, G)=C_{0}(K, G)$ consisting of those zero chains $\sum g_{i} \sigma_{0}^{4}$ for which $\sum g_{i}=0$. Since $K$ is augmentable it follows that $B_{0}(K, G) \subset \bar{Z}_{0}(K, G)$. Define $\bar{H}_{0}(K, G)$ $=\bar{Z}_{0}(K, G) / B_{0}(K, G)$. Clearly $\bar{H}_{0}(K, G)$ is a subgroup of $H_{0}(K, G)$ $=Z_{0}(K, G) / B_{0}(K, G)$.

Let $\bar{B}^{0}(K, G)$ be the subgroup of $C^{0}(K, G)$ consisting of all cochains $f \in C^{0}(K, G)$ for which $f\left(\sigma_{0}\right)=$ const. Since $K$ is augmentable every such cochain is a cocycle and $\bar{B}^{0}(K, G) \subset Z^{0}(K, G)$. Define $\bar{H}^{0}(K, G)=Z^{0}(K, G) / \bar{B}^{0}(K, G)$. Clearly $\bar{H}^{0}(K, G)$ is a factor group of the ordinary cohomology group $H^{0}(K, G)=Z^{0}(K, G)$.

If $\tau: K_{1} \rightarrow K_{2}$ is a chain transformation and both complexes $K_{1}$ and $K_{2}$ are augmentable then $\tau$ is said to be augmentable provided $I(c)=0$ implies $I(\tau c)=0$ for every zero-chain $c$ in $K_{1}$. It is easy to see that $\tau$ is augmentable if and only if it can be extended to a chain transformation of the augmented complexes. If $\tau$ is augmentable then it maps the reduced homology groups of $K_{1}$ into those of $K_{2}$ and the reduced cohomology groups of $K_{2}$ into those of $K_{1}$.

The abstract complexes $k(P), K(P)$, and $S(X)$ considered so far were all augmentable. Similarly, all the chain transformations defined in this chapter were augmentable. Consequently the chain transformation $\alpha: K(P) \rightarrow k(P)$ and $\beta: K(P) \rightarrow S(P)$ define homomorphisms of the reduced homology and cohomology groups, which become isomorphisms under the conditions of Corollary 10.2.

It can easily be seen that for any nontrivial coefficient group $G$ either of the conditions $\bar{H}_{0}(X, G)=0$ or $\bar{H}^{0}(X, G)=0$ is equivalent with the arcwise connectedness of the space $X$.

12. Homologies and cohomologies in a group. Let $W$ be a discrete group written multiplicatively. In [5], [6], and [7], homology and cohomology groups of $W$ were defined and various applications were given. It will be shown here that these groups fit into the pattern of the equivariant and other groups studied in this paper.

We define an abstract complex $K_{W}$, associated with the group $W$, as follows. The $q$-cells of $K_{W}$ are ordered $(q+1)$-tuples $\left(w_{0}, \cdots, w_{q}\right)$ of elements of $W$ (with possible repetitions). The boundary is defined for $q>0$ as

$$
\partial\left(w_{0}, \cdots, w_{q}\right)=\sum_{i=0}^{q}(-1)^{i}\left(w_{0}, \cdots, w_{i}^{\widehat{y}}, \cdots, w_{q}\right) .
$$


Since $\partial \partial=0, K_{W}$ is an augmentable closure finite abstract complex in the sense of $\$ 1$. Given $w \in W$ we define

$$
w\left(w_{0}, \cdots, w_{q}\right)=\left(w w_{0}, \cdots, w w_{q}\right) .
$$

This gives a chain transformation $w: K_{W} \rightarrow K_{W}$ so that $W$ is defined as a group of operators for the complex $K_{W}$.

$$
\begin{array}{lll}
\bar{H}_{0}\left(K_{W}, G\right)=0, & H_{q}\left(K_{W}, G\right)=0 & \text { for } q>0, \\
\bar{H}^{0}\left(K_{W}, G\right)=0, & H^{q}\left(K_{W}, G\right)=0 & \text { for } q>0 .
\end{array}
$$

Given a $q$-cell $\sigma_{q}=\left(w_{0}, \cdots, w_{q}\right)$, define $D \sigma_{q}=\left(1, w_{0}, \cdots, w_{q}\right)$. For $c=\sum g_{i} \sigma_{q}^{s}$ define $D c=\sum g_{i} D \sigma_{q}^{s}$. Clearly $D$ is a homomorphism $D: C_{q}\left(K_{W}, G\right)$ $\rightarrow C_{q+1}\left(K_{W}, G\right)$. From the definition of $D$ it follows that $\partial D c=c-D \partial c$ for $q>0$. Hence if $c \in Z_{q}\left(K_{W}, G\right)$ then $D \partial c=0$ and $c=\partial D c$ which implies $c \in B_{q}\left(K_{W}, G\right)$. If $c \in \bar{Z}_{0}\left(K_{W}, G\right)$ then $c=\partial D c$ and $c \in B_{0}\left(K_{W}, G\right)$. This proves (12.1). The proof of (12.2) is obtained by defining $\left(D^{*} f\right)(c)=f(D c)$ for every cochain $f$.

$$
\begin{array}{ll}
H_{q}^{e}\left(K_{W}, G\right) \approx H_{q-1}^{r}\left(K_{W}, G\right) & \text { for } q>1, \\
H_{e}^{q}\left(K_{W}, G\right) \approx H_{r}^{q-1}\left(K_{W}, G\right) & \text { for } q>1 .
\end{array}
$$

In fact, in the exact sequence (2.2) for $K_{W}$ we have

$$
H_{q-1}\left(K_{W}, G\right) \leftarrow H_{q-1}^{r}\left(K_{W}, G\right) \underset{\partial}{\leftarrow} H_{q}^{e}\left(K_{W}, G\right) \leftarrow H_{q}\left(K_{W}, G\right) .
$$

For $q>1$ the two outside groups are trivial, hence $\partial: H_{q}^{e}\left(K_{W}, G\right) \rightarrow H_{q-1}^{r}\left(K_{W}, G\right)$ is an isomorphism of the first group onto the second one. For cohomologies the proof is analogous.

Propositions (12.1)-(12.4) show that the ordinary homologies and cohomologies are trivial, and that the equivariant ones determine the residual ones. Consequently we adopt the following definitions.

Given any discrete abelian group $G$ with $W$ as right operators we define the $q$ th homology group $H_{q}(W, G)$ of $W$ over $G$ to be the equivariant homology group $H_{q}^{s}\left(K_{W}, G\right)$ of $K_{W}$ over $G$.

Given any topological abelian group $G$ with $W$ as left operators we define the $q$ th cohomology group $H^{q}(W, G)$ of $W$ over $G$ to be the equivariant cohomology group $H_{e}^{q}\left(K_{W}, G\right)$ of $K_{W}$ over $G$.

The cohomology group $H^{q}(W, G)$ can be given a direct and rather simple description. A $q$-cochain $f \in C^{q}\left(K_{W}, G\right)$ is a function $f$ of $q+1$ variables in $W$ with values in $G$

$$
f\left(w_{0}, \cdots, w_{q}\right) \in G .
$$

The equivariance condition on $f$ reads

$$
w f\left(w_{0}, \cdots, w_{q}\right)=f\left(w w_{0}, \cdots, w w_{0}\right) .
$$

The coboundary $\delta f$ is defined by the formula 


$$
(\delta f)\left(w_{0}, \cdots, w_{q+1}\right)=\sum_{i=0}^{q+1}(-1)^{i} f\left(w_{0}, \cdots, w_{i}, \cdots, w_{q+1}\right) .
$$

If we denote the group of equivariant cochains by $C^{q}(W, G)$, the subgroup of equivariant cocycles by $Z^{q}(W, G)$, and the coboundaries of equivariant $(q-1)$-cochains by $B^{q}(W, G)$, then $H^{q}(W, G)=Z^{q}(W, G) / B^{q}(W, G)$. With this description we find that the cohomology groups $H^{q}(W, G)$ coincide with those studied by Eilenberg and MacLane [5, 6, 7].

13. Comparison with the homology groups of Hopf. In [10] Hopf has defined homology groups $W_{G}^{q}$ where $W$ is a discrete multiplicative group and $G$ is a ring with a unity element. These groups were invented for the same purpose as the groups defined in [5] and [6]. It will be shown here that the Hopf groups $W_{q}^{q}$ are isomorphic with the homology groups $H_{q}(W, G)$. The restriction that $G$ is a ring and that $W$ does not operate on $G$ (or, what amounts to the same, that $W$ operates simply on $G$ ) is maintained.

We take over without modification the concepts and propositions established in $\S \S 1$ and 2 of Hopf's paper [10, pp. 40-47]. In particular $P$ will be the group ring of $W$ over $G, S$ is the ring homomorphism $P \rightarrow G$ defined by $S\left(\sum g_{i} w_{i}\right)=\sum g_{i}, P_{0}$ is the two-sided ideal defined by $S$. The ring $G$ is converted in to a $P$-module by setting $p g=S(p) g$.

We now turn to the chains in the complex $K_{W}$. We shall use the following abbreviations: $C_{q}=C_{q}\left(K_{W}, G\right), Z_{q}=Z_{q}\left(K_{W}, G\right), B_{q}=B_{q}\left(K_{W}, G\right)$, and similarly for the residual groups $C_{q}^{r}, Z_{q}^{r}$ and $B_{q}^{r}$.

Given a chain $c=\sum g_{i} q^{i} \in C_{q}$ and given $p=\sum g_{j} w_{j} \in P$, define $p c=\sum_{i, j} g_{i} g_{j}\left(w_{j} \sigma_{q}^{i}\right)$. With this definition $C_{q}$ becomes a $P$-module.

(13.1) $C_{q}$ is a free P-module.

Indeed, every $q$-cell $\sigma_{q}$ of $K_{W}$ can uniquely be written as $\sigma_{q}=w \bar{\sigma}_{q}$ where $\bar{\sigma}_{q}$ has the form $\bar{\sigma}_{q}=\left(1, w_{1}, \cdots, w_{q}\right)$. Consequently, if we identify the symbols $\sigma_{q}$ and $e \sigma_{q}$, where $e$ is the unity element of $G$, it follows that $\left\{\bar{\sigma}_{q}\right\}$ is a free $P$-basis of $C_{q}$.

Consider the homomorphism $\partial: C_{q+1} \rightarrow B_{q}$. The kernel of $\partial$ is $Z_{q+1}$. Since, by (12.1), $Z_{q+1}=B_{q+1}$ and since $\partial(p c)=p \partial c$ it follows that

(13.2) $\partial$ is a P-homomorphism mapping $C_{q+1}$ onto $B_{q}$ with $B_{q+1}$ as kernel. In addition we define a $P$-homomorphism $I: C_{0} \rightarrow G$ by setting $I\left(\sum g_{i} \sigma_{0}^{s}\right)$ $=\sum g_{i}$.

(13.3) $I$ is a $P$ homomorphism mapping $C_{0}$ onto $G$ with $B_{0}$ as kernel.

Propositions (13.1)-(13.3) imply that

$$
\left\{G, C_{0} \subset B_{0}, \cdots, C_{q} \subset B_{q}, \cdots\right\}
$$

is a $\{G, P\}$ sequence in the sense of Hopf $[10$, p. 42$]$. The groups $W_{G}^{q}$ are then defined by

$$
W_{G}^{q+1}=B_{q} \cap\left[C_{q}\right]_{0} /\left[B_{q}\right]_{0}
$$


where $\left[C_{q}\right]_{0}$ (or $\left.\left[B_{q}\right]_{0}\right)$ is the set of all chains of the form $\sum p_{i} c_{i}$ where $p_{i} \in P_{0}$ and $c_{i} \in C_{q}$ (or $c_{i} \in B_{q}$ ).

(13.5) $\left[C_{q}\right]_{0}=C_{q}^{r}$ and $\left[B_{q}\right]_{0}=B_{q}^{r}$.

In fact, $C_{q}^{r}$ is generated by all chains of the form $g w c-g c=p c$ where $p=g w-g$. Since $S(p)=g-g=0$ it follows that $C_{q}^{+} \subset\left[C_{q}\right]_{0}$. Conversely $\left[C_{q}\right]_{0}$ is generated by chains of the form $c=p d, p \in P_{0}, d \in C_{q}$. Let $p=\sum g_{i} w_{i}$, then $\sum g_{i}=0$ and $c=\sum\left(g_{i} w_{i}-g_{i}\right) d$. This shows that $c \in C_{q}^{r}$. Every element $c \in\left[B_{q}\right]_{0}$ is of the form $c=\sum p_{i} \partial c_{i}$ with $p_{i} \in P_{0}$. Hence $c=\partial \sum p_{i} c_{i}$ and since $\sum p_{i} c_{i}$ $\in\left[C_{q+1}\right]_{0}=C_{q+1}^{r}$ it follows that $c \in B_{q}^{r}$. Conversely if $c \in B_{q}^{r}$ then $c=\partial d$ with $d \in C_{q+1}=\left[C_{a+1}^{r}\right]_{0}$. Hence $d=\sum p_{i} d_{i}$ with $p_{i} \in P_{0}$ and $c=\partial d=\sum p_{i} \partial d_{i}$ which shows that $c \in\left[B_{q}\right]_{0}$.

Combining (13.4) and (13.5) gives

$$
W_{G}^{q+1}=B_{q} \cap C_{q}^{r} / B_{q}^{r} \text {. }
$$

We are now able to prove the main result of this section

$$
W_{G}^{q+1} \approx H_{q+1}(W, G) \text {. }
$$

First if $q>0$ then $B_{q}=Z_{q}$ in virtue of (12.1). Consequently $W_{q}^{q+1}=B_{q}$ $\cap C_{q}^{r} / B_{q}^{r}=Z_{q} \cap C_{q}^{r} / B_{q}^{r}=Z_{q}^{r} / B_{q}^{r}=H_{q}^{r}(W, G)$. Thus (13.7) is a consequence of (12.3). If $q=0$ we examine the homomorphisms

$$
H_{0}\left(K_{W}, G\right) \underset{\phi}{\leftarrow} H_{0}^{r}\left(K_{W}, G\right) \underset{\partial}{\leftarrow} H_{1}^{e}\left(K_{W}, G\right) \underset{\psi}{\leftarrow} H_{1}\left(K_{W}, G\right)
$$

The kernel of $\phi$ is the group $B_{0} \cap Z_{0}^{r} / B_{0}^{r}=B_{0} \cap C_{0}^{r} / B_{0}^{r}=W_{\sigma}^{1}$. Hence by exactness $W_{G}^{1}$ is the image of $\partial$. But since $H_{1}\left(K_{w}, G\right)=0, \partial$ is an isomorphism and $H_{1}\left(K_{w}, G\right) \approx W_{G}^{1}$. Hence $W_{G}^{1} \approx H_{1}(W, G)$.

\section{Chapter III. RElations BETWEen THE HOMOLOGY THEORY OF SPACES AND GROUPS}

14. The basic chain transformation. Let $X$ be a topological space with a group $W$ as operators. The $W$-transforms of every point $x$ in $X$ form a subset of $X$ called an orbit. The orbits are disjoint. A set $\{\bar{x}\}$ containing one point out of each orbit will be called a set of representatives.

We shall assume that $W$ operates without fixed points, that is, that $w x \neq x$ for each $x$ and each $w \neq 1$. In this case every $x \in X$ can uniquely be written as $x=w \bar{x}$ where $\bar{x}$ is a representative.

Consider a singular simplex $T: s \rightarrow X$ where $s=\left\langle p_{0} \cdots p_{q}\right\rangle$ is a euclidean simplex with ordered vertices $p_{0}<\cdots<p_{q}$. The vertices $T\left(p_{0}\right), \cdots, T\left(p_{q}\right)$ of $T$ can uniquely be written as $w_{0} \bar{x}_{0}, \cdots, w_{q} \bar{x}_{q}$ where $\bar{x}_{0}, \cdots, \bar{x}_{q}$ are elements of a fixed set of representatives $\{\bar{x}\}$. Define $\tau T$ to be the $q$-cell of $K_{W}$ determined by $w_{0}, \cdots, w_{q}$

$$
\tau T=\left(w_{0}, \cdots, w_{q}\right) .
$$


This maps the singular simplexes in $X$ into cells of $K_{W}$, and defines homomorphisms $\tau: C_{q}(X) \rightarrow C_{q}\left(K_{W}\right)$. It is obvious from the definition that $\tau$ and $\partial$ commute. Hence $\tau$ is a chain transformation

$$
\tau: S(X) \rightarrow K_{W} .
$$

It is clear that $\tau$ is augmentable. Moreover $\tau$ is equivariant since $\tau(w T)$ $=\left(w w_{0}, \cdots, w w_{q}\right)=w(\tau T)$.

We emphasize that $\tau$ has been constructed relative to a particular choice of representatives $\{\bar{x}\}$. A different set of representatives would lead to a different chain transformation.

15. Acyclic spaces. An augmentable complex $K$ is said to be acyclic in dimensions less than $q(q>0)$ provided

$$
\bar{H}_{0}(K)=0, \quad H_{i}(K)=0 \quad \text { for } 0<i<q .
$$

(12.1) implies that the complex $K_{W}$ is acyclic in all dimensions. We shall assume here that the space $X$ is acyclic in dimensions less than $q$ (that is, that the complex $S(X)$ is acyclic in dimensions less than $q$ ). Under this assumption we shall construct three sequences of homomorphisms

$$
\begin{array}{rlrl}
\rho: C_{i}\left(K_{W}\right) & \rightarrow C_{i}(X), & i=0,1, \cdots, q, \\
D_{1}: C_{i}(X) & \rightarrow C_{i+1}(X), & i=0,1, \cdots, q-1, \\
D_{2}: C_{i}\left(K_{W}\right) \rightarrow C_{i+1}\left(K_{W}\right), & i=0,1, \cdots, q,
\end{array}
$$

such that, for all $w \in W$,

$$
w \rho=\rho w, \quad w D_{1}=D_{1} w, \quad w D_{2}=D_{2} w
$$

and that

$$
\begin{aligned}
\partial \rho c & =\rho \partial c, \\
\partial D_{1} c & =c-\rho \tau c-D_{1} \partial c, \\
\partial D_{2} c & =c-\tau \rho c-D_{2} \partial c,
\end{aligned}
$$

for all chains $c$ (in $X$ or in $K_{W}$ as the case may be) for which the operators $\rho, D_{1}$ and $D_{2}$ are defined. For the dimension zero the last terms in (15.6) and (15.7) are to be omitted and (15.5) is void.

For the construction it will be convenient to use the concept of a basic cell in $K_{W}$ and in $X$. A cell $\sigma_{q}$ in $K_{W}$ will be called basic if its first vertex is the unit element of $W$, that is, if $\sigma_{q}=\left(1, w_{1}, \cdots, w_{q}\right)$. A simplex $T$ in $X$ will be called basic if its leading (0th) vertex is one of the points $\bar{x}$. Clearly every cell in $K_{W}$ or $X$ can be represented uniquely as a $W$-transform of a basic cell. All the homomorphisms $\rho, D_{1}$ and $D_{2}$ will be defined for basic cells first and then be extended to all other cells by means of (15.4).

We define $\rho(1)$, where (1) is the basic zero cell of $K_{W}$, to be any point $\bar{x}$ (treated as a zero cell of $X$ ). By (15.4) the definition is extended to all 0 -cells 
of $K_{W}$. From now on, proceed by induction. Assume that $\rho$ has been defined for all $j$-cells for $j<i \leqq q$ and that it satisfies (15.4) and (15.5). Given any basic $i$-cell $\sigma_{i}$ of $K_{W}$ the chain $\rho \partial \sigma_{i}$ is an integral chain in $X$ of dimension $i-1$ and since $(15.5)$ is satisfied it is a cycle in $X$. Since $i-1<q, H_{i-1}(X)=0$ and there is an $i$-chain in $X$ that we shall denote by $\rho \sigma_{i}$ such that $\partial \rho \sigma_{i}=\rho \partial \sigma_{i}$. Again using (15.4) we extend the definition of $\rho$ to non-basic cells. For $j=1$ the argument differs slightly.

Next we proceed with the definition of $D_{1}$. Given a basic 0 -cell $\bar{x}$ in $X$, consider the 0 -cycle $\bar{x}-\rho \tau \bar{x}$. Since $\rho \tau \bar{x}=\rho(1)$ is also a single point of $X$ and since $X$ is arcwise connected $\left(\bar{H}_{0}(X)=0\right)$ there is an integral 1-chain $D_{1} \bar{x}$ such that $\partial D_{1} \bar{x}=\bar{x}-\rho \tau \bar{x}$. The definition of $D_{1}$ is then extended to the non-basic 0 -cells of $X$ by means of (15.4). From now on, proceed by induction. Assume that $D_{1}$ has been defined for all $j$-cells for $j<i<q$ and that (15.4) and (15.6) hold. Given any basic $i$-simplex $T$ of $X$ consider the $i$-chain in $X$

$$
c=T-\rho \tau T-D_{1} \partial T \text {. }
$$

Since $\quad \partial c=\partial T-\partial \rho \tau T-\partial D_{1} \partial T=\partial T-\rho \tau \partial T-\left(\partial T-\rho \tau \partial T-D_{1} \partial \partial T\right)=0, \quad$ the chain $c$ is an $i$-cycle in $X$ and since $i<q, H_{i}(X)=0$ and there is an $(i+1)$-chain $D_{1} T$ such that $\partial D_{1} T=c$. Having thus defined $D_{1} T$ for basic $T$ 's we use (15.4) to extend the definition to all the $i$-simplexes of $X$.

The definition of $D_{2}$ is quite similar. It can be carried one dimension further than that of $D_{1}$ since $H_{q}\left(K_{W}\right)=0$.

16. The basic isomorphisms. We are now in a position to formulate the main theorem of this chapter.

THEOREM 16.1. Given a topological space $X$ acyclic in dimensions less than $q$ and given a group $W$ operating on the space $X$ without fixed points, the following isomorphisms hold.

For homology groups over a discrete abelian group $G$ with $W$ as a group of right operators:

$$
\begin{aligned}
& H_{i}^{e}(X, G) \approx H_{i}(W, G), \quad i=1, \cdots, q-1 \text {, } \\
& H_{i}^{r}(X, G) \approx H_{i+1}(W, G), \quad i=1, \cdots, q-1, \\
& H_{q}^{e}(X, G) / \psi H_{q}(X, G) \approx H_{q}(W, G),
\end{aligned}
$$

where $\psi$ is the homomorphism $\psi: H_{q}(X, G) \rightarrow H_{q}^{e}(X, G)$ from the exact sequence (2.2).

For cohomology groups over a topological abelian group $G$ with $W$ as a group of left operators:

$$
\begin{array}{ll}
H_{e}^{i}(X, G) \approx H^{i}(W, G), & i=1, \cdots, q-1, \\
H_{r}^{i}(X, G) \approx H^{i+1}(W, G), & i=1, \cdots, q-1, \\
K^{q}(X, G) \approx H^{q}(W, G), &
\end{array}
$$


where $K^{q}(X, G)$ is the kernel of the homomorphism $\phi: H_{e}^{q}(X, G) \rightarrow H^{q}(X, G)$ from the exact sequence (4.2).

Proof. The chain transformation $\tau: X \rightarrow K_{W}$ induces homomorphisms for all $i \geqq 0$

$$
\tau_{i}: H_{i}(X, G) \rightarrow H_{i}\left(K_{W}, G\right), \quad \tau^{i}: H^{i}\left(K_{W}, G\right) \rightarrow H^{i}(X, G) .
$$

Since $\tau$ commutes with $W$ similar homomorphisms hold for the equivariant and for the residual groups.

$$
\begin{array}{rlrl}
\tau_{i}^{e}: H_{i}^{e}(X, G) & \rightarrow H_{i}^{e}\left(K_{W}, G\right), & \tau_{e}^{i}: H_{e}^{i}\left(K_{W}, G\right) \rightarrow H_{e}^{i}(X, G), \\
\tau_{i}^{r}: H_{i}^{r}(X, G) \rightarrow H_{i}^{r}\left(K_{W}, G\right), & \tau_{r}^{i}: H_{r}^{i}\left(K_{W}, G\right) \rightarrow H_{r}^{i}(X, G) .
\end{array}
$$

All the isomorphisms formulated in Theorem 16.1 will be established by means of the homomorphisms (16.8) and (16.9). For the dimensions $i<q$ we have the "inverse" chain transformation $\rho: K_{W} \rightarrow X$ and the operator $D_{1}$ showing that $\rho \tau$ is chain homotopic to the identity map $1: X \rightarrow X$ and the operator $D_{2}$ showing that $\tau \rho$ is chain homotopic to $1: K_{W} \rightarrow K_{W}$. Since $\rho, D_{1}$, and $D_{2}$ are equivariant we conclude following $\$ 5$ that for the dimensions $i<q$ all the homomorphisms (16.7), (16.8), and (16.9) are isomorphisms onto. From (16.8) we deduce (16.1) and (16.4), from (16.9), (12.3), and (12.4) we deduce (16.2) and (16.5). From (16.7), (12.1), and (12.2) we deduce $H_{i}(X, G)=0$, $H^{i}(X, G)=0$ for $1<i<q$. In order to prove (16.3) we study the diagram

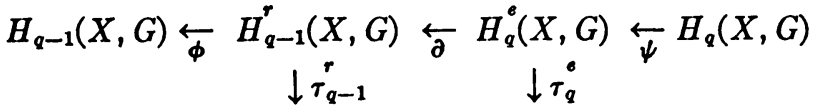

$$
\begin{aligned}
& H_{q-1}\left(K_{W}, G\right) \underset{\phi_{W}}{\leftarrow} H_{q-1}^{r}\left(K_{W}, G\right) \underset{\partial_{W}}{\leftarrow} H_{q}^{e}\left(K_{W}, G\right) \underset{\psi_{W}}{\leftarrow} H_{q}\left(K_{W}, G\right)
\end{aligned}
$$

which is part of the mapping of the exact lower sequence of $X$ over $G$ in to that of $K_{W}$ over $G$ furnished by the chain transformation $\tau$. Since $H_{q-1}(X, G)$ $=0, \partial$ is a mapping onto. Since $H_{q-1}\left(K_{W}, G\right)=H_{q}\left(K_{W}, G\right)=0$ the mapping $\partial_{W}$ is an isomorphism onto. We have already shown that $\tau_{q-1}^{r}$ is an isomorphism onto. Since $\tau_{q-1}^{r} \partial=\partial_{W} \tau_{a}^{e}$ and both $\partial_{W}$ and $\tau_{q-1}^{r}$ are isomorphisms it follows $\tau_{q-1}^{r}$ is a mapping onto and that $\partial$ and $\tau_{q}^{e}$ have the same kernel. But the kernel of $\partial$ is the image group $\psi H_{q}(X, G)$, hence this last group is the kernel of $\tau_{q}^{e}$. This proves (16.3).

The isomorphism (16.6) follows from a similar diagram for cohomologies

$$
\begin{aligned}
& H^{q-1}(S, G) \underset{\psi}{\rightarrow} B_{r}^{q-1}(X, G) \underset{\delta}{\rightarrow} H_{e}^{q}(X, G) \underset{\phi}{\rightarrow} H^{q}(X, G) \\
& \uparrow \tau_{r}^{q-1} \quad \tau_{\tau}^{q} \\
& H^{q-1}\left(K_{W}, G\right) \underset{\psi_{W}}{\longrightarrow} H_{r}^{q-1}\left(K_{W}, G\right) \underset{\delta_{W}}{\longrightarrow} H_{e}^{q}\left(K_{W}, G\right) \underset{\phi_{W}}{\longrightarrow} H^{q}\left(K_{W}, G\right) .
\end{aligned}
$$

Since $H^{q-1}\left(K_{W}, G\right)=H^{q}\left(K_{W}, G\right)=0, \delta_{W}$ is an isomorphism onto. Since 
$H^{q-1}(X, G)=0, \delta$ is an isomorphism into. Since both $\tau_{r}^{q-1}$ and $\delta_{W}$ are isomorphisms onto and $\delta_{W} \tau_{e}^{q}=\tau_{r}^{q-1} \delta$ it follows that $\tau_{e}^{q}$ is an isomorphism into and has the same image as $\delta$. But the image of $\delta$ is the kernel of $\phi$ that we have denoted by $K_{\bullet}^{\bullet}(X, G)$. Hence we have (16.6).

17. Residual groups. The previous discussion was incomplete inasmuch as we didn't analyze the homomorphisms

$$
\tau_{q}^{r}: H_{q}^{r}(X, G) \rightarrow H_{q}^{r}\left(K_{W}, G\right), \quad \tau_{r}^{q}: H_{r}^{q}\left(K_{W}, G\right) \rightarrow H_{r}^{q}(X, G) .
$$

The discussion requires the introduction of new subgroups of the residual groups.

Let $K$ be any complex with operators $W$. Given any subgroup $A$ of $C_{q}(K)$, denote by $[A] \mathrm{r}$ the subgroup of $C_{q}(K, G)$ generated by elements of the form $g(w a)-(g w) a$ for $g \in G, w \in W$ and $a \in A$. As usual we assume that $W$ operates on $G$ on the right. We have

$$
C_{q}^{r}(K, G)=\left[C_{q}(K)\right]^{r}, \quad B_{q}^{r}(K, G)=\left[B_{q}(K)\right]^{r} .
$$

The first of these equalities is the definition of $C_{q}^{r}$; the other follows from the relation $\partial[g(w c)-(g w) c]=g(w \partial c)-(g w) \partial c$.

Consider the group $\left[Z_{q}(K)\right]^{r}$. Clearly

$$
B_{q}^{r}(K, G) \subset\left[Z_{q}(K)\right]^{r} \subset Z_{q}^{r}(K, G) .
$$

Hence $\left[Z_{q}(K)\right]^{r} / B_{q}^{r}(K, G)$ is a subgroup of $H_{q}^{r}(K, G)$. We shall denote this subgroup by $D_{q}^{r}(K, G)$. If $H_{q}(K)=0$ then $B_{q}(K)=Z_{q}(K)$ and $B_{q}^{r}(K, G)$ $=\left[B_{q}(K)\right]^{r}=\left[Z_{q}(K)\right]^{r}$ so that:

(17.3) If $H_{q}(K)=0$ then $D_{q}^{r}(K, G)=0$.

Let now $G$ be a topological abelian group with $W$ as a group of left operators. For a subgroup $A$ of $C_{q}(K)$ we define $[A]_{0}$ to be the set of all cochains $f \in C^{q}(K, G)$ such that $f(w a)=w f(a)$ for all $w \in W$ and all $a \in A$. It can easily be seen that

$$
C_{e}^{q}(K, G)=\left[C_{q}(K)\right]_{e}, \quad Z_{r}^{q}(K, G)=\left[B_{q}(K)\right]_{e} .
$$

Consider the group $\left[Z_{q}(K)\right]_{e} ;(17.4)$ implies that

$$
B_{r}^{q}(K, G) \subset\left[Z_{q}(K)\right]_{\bullet} \subset Z_{r}^{q}(K, G) .
$$

Consequently $\left[Z_{q}(K)\right]_{e} / B_{r}^{q}(K, G)$ is a subgroup of $H_{r}^{q}(K, G)$; we denote this subgroup by $D_{r}^{q}(K, G)$. If $H_{q}(K)=0$ then $Z_{q}(K)=B_{q}(K)$ and $\left[Z_{q}(K)\right]_{\text {e }}$ $=Z_{r}^{q}(K, G)$. Consequently:

(17.5) If $H_{q}(K)=0$ then $D_{r}^{q}(K, G)=H_{r}^{q}(K, G)$.

We shall now prove that:

(17.6) $\tau_{i}^{r}$ maps $D_{i}^{r}(X, G)$ into the zero of $H_{i}^{r}\left(K_{W}, G\right)$ for all $i>0$.

In fact, $D_{i}^{r}(X, G)$ is mapped into $D_{i}^{r}\left(K_{W}, G\right)$ which is zero in virtue of (12.1) and (17.3). 
(17.7) $\tau_{r}^{i}$ maps $H_{r}^{i}\left(K_{W}, G\right)$ into the group $D_{r}^{i}(X, G)$ for $i>0$.

In fact, $\tau_{r}^{i}$ maps $D_{r}^{i}\left(K_{W}, G\right)$ into $D_{r}^{i}(X, G)$, but $H_{r}^{i}\left(K_{W}, G\right)=D_{r}^{i}\left(K_{W}, G\right)$ in virtue of (12.2) and (17.5).

AdDENDUM to THEOREM 16.1. Under the conditions of Theorem 16.1 the following isomorphisms hold:

$$
\begin{aligned}
H_{q}^{r}(X, G) / D_{q}^{r}(X, G) & \approx H_{q+1}(W, G), \\
D_{r}^{q}(X, G) & \approx H^{q+1}(W, G) .
\end{aligned}
$$

In virtue of (12.3) and (12.4) the proposition is a consequence of the following two propositions:

(17.10) Under the assumptions of Theorem $16.1, \tau_{q}^{r}$ maps $H_{a}^{r}(X, G)$ onto the group $H_{a}^{r}\left(K_{W}, G\right)$ with the group $D_{a}^{r}(K, G)$ as kernel.

(17.11) Under the assumptions of Theorem 16.1, $\tau_{r}^{q}$ maps $H_{r}^{q}\left(K_{W}, G\right)$ isomorphically onto the subgroup $D_{r}^{q}(X, G)$ of $H_{r}^{q}(X, G)$.

We shall prove only (17.10). The proof of (17.11) is analogous.

Let $c \in Z_{q}^{r}\left(K_{W}, G\right)$, that is, let $c \in C_{q}^{r}\left(K_{W}, G\right)$ and $\partial c=0$. Since $\rho$ commutes with $W$ and $\partial$ we have $\rho c \in C_{q}^{r}(X, G)$ and $\partial \rho c=0$ so that $\rho c \in Z_{a}^{r}(X, G)$. We have $c-\tau(\rho c)=\partial D_{2} c-D_{2} \partial c=\partial D_{2} c$. Since $D_{2}$ commutes with $W, D_{2} c$ $\in C_{q+1}^{r}\left(K_{W}, G\right)$ so that $c \equiv \tau(\rho c) \bmod B_{q}^{r}\left(K_{W}, G\right)$. This proves that $\tau_{i}^{r}$ is a mapping onto.

Given any $q$-chain $c$ in $C_{q}(X)$ or in $C_{q}(X, G)$, define

$$
R c=c-\rho \tau c-D_{1} \partial c .
$$

We have $R c=\partial c-\partial \rho \tau c-\partial D_{1} \partial c=\partial c-\rho \tau \partial c-\left(\partial c-\rho \tau \partial c-D_{1} \partial \partial c\right)=0$ so that $R c$ is always a cycle. If $c=g(w d)-(g w) d$ then, since $R$ and $W$ commute, $R c=g(w R d)-(g w) R d$ and since $R d$ is a cycle this shows that $R c \in\left[Z_{q}(X)\right]$. Suppose now that $c \in Z_{q}^{r}(X, G)$ and that $\tau c \in B_{q}^{r}\left(K_{W}, G\right)$, then $\partial c=0$, $\rho(\tau c) \in B_{q}^{r}(X, G)$ and $R c \in\left[Z_{q}(X)\right] r$. But $c=R c+\rho \tau c+D_{1} \partial c=R c+\rho \tau c$ $\in\left[Z_{q}(X)\right]^{r}+B_{q}^{r}(X)=\left[Z_{q}(X)\right] r$. This proves that $D_{q}^{r}(X, G)$ contains the ker-. nel of $\tau_{q}^{r}$. Since by (17.6) the kernel contains $D_{q}^{r}(X, G)$, proposition (17.10) is proven.

18. Discussion of coefficients. In all of our discussion so far the group of integers was given preferential treatment, the chain transformations and homotopies were defined for in tegral chains and then carried over to arbitrary chains and cochains. We shall now see to what extent the group of integers can be replaced by an arbitrary group.

Let $J$ be a discrete abelian group. Given any discrete abelian group $G$ we define $G_{J}$ to be the tensor product $\left.{ }^{4}\right) G \circ J$. If a group $W$ operates on $G$ (on the right), we define operators for $G_{J}$ by setting $(g j) w=(g w) j$ for every generator $g j \in G_{J}$. If $J$ is the group of integers than $G_{J} \approx G$.

(4) See footnote 2 . 
Given a topological abelian group $G$ we define $G^{J}$ to be the group $\operatorname{Hom}(J, G)$ with the topology defined as in $\S 3$. If $W$ operates on $G$ (on the left) we define operators for $G^{J}$ by setting $(w \phi)(j)=w(\phi(j))$ for every $\phi \in G^{J}$. If $J$ is the group of integers then $G^{J} \approx G$.

Let now $K$ be an abstract complex with operators $W$. Since the tensor product is associative we have

$$
C_{q}\left(K, G_{J}\right)=G_{J} \circ C_{q}(K)=G \circ\left(J \circ C_{q}(K)\right)=G \circ C_{q}(K, J) .
$$

Thus the chains in $K$ with coefficients in $G_{J}$ can be written in the form $\sum g_{i} c_{i}$ where $g_{i} \in G, c_{i} \in C_{q}(K, J)$. It is easy to see that the group of residual chains $C_{a}^{T}\left(K, G_{J}\right)$ is generated by elements of the form $g(w c)-(g w) c$.

For the groups of cochains we have

$$
\begin{aligned}
C^{q}\left(K, G^{J}\right) & =\operatorname{Hom}\left(C_{q}(K), \operatorname{Hom}(J, G)\right) \approx \operatorname{Hom}\left(J \circ C_{q}(K), G\right) \\
& =\operatorname{Hom}\left(C_{q}(K, J), G\right) .
\end{aligned}
$$

The middle isomorphism is a special case of the general natural isomor$\operatorname{phism}\left({ }^{(5)} \operatorname{Hom}(J \circ C, G) \approx \operatorname{Hom}(C, \operatorname{Hom}(J, G))\right.$ valid for any three abelian groups $J$ (discrete), $C$ (discrete), and $G$ (topological). We may thus consider every cochain $f \in C^{q}\left(K, G^{J}\right)$ as a homomorphism $f: C_{q}(K, J) \rightarrow G$. It is easy to see that the group of equivariant cochains $C_{e}^{r}\left(K, G^{J}\right)$ consists of those homomorphisms $f$ satisfying the condition $w f(c)=f(w c)$ for each $c \in C_{q}(K, J)$ and $w \in W$.

The concepts of chain transformation and of chain homotopy can now be generalized to chain transformations over $J$ and chain homotopies over $J$. An ordinary chain transformation induces mappings of the homology and cohomology groups with any coefficients. A chain transformation over $J$ induces homomorphisms of the homology groups with coefficients $G_{J}$ and of the cohomology groups with coefficients $G^{J}$.

We now assume that $J$ is a ring with a unit element. Every chain over $J$ can then be multiplied on the left by elements of $J$, so that the groups $C_{q}(K, J)$ are converted into $J$-moduls. The boundary operator is then a $J$-homomorphism.

Let $X$ be a topological space with $W$ operating on $X$ without fixed points. The homomorphism

$$
\tau: C_{q}(X, J) \rightarrow C_{q}\left(K_{W}, J\right)
$$

is then a chain transformation over $J$. Moreover each $\tau$ is a $J$-homomorphism. If we further assume that $X$ is acyclic over $J$ in dimensions less than $q$, that is, that $\bar{H}_{0}(X, J)=0, H_{i}(X, J)=0$ for $0<i<q$, then the constructions of $\$ 15$ carry over without change provided that we replace integral chains by chains over $J$ and require $\rho, D_{1}$ and $D_{2}$ to be $J$-homomorphisms. The arguments of $\$ 16$ and $\$ 17$ carry over provided that homologies are taken over $G_{J}$ and cohomologies over $G^{J}$.

(5) See S. Eilenberg and S. MacLane, Proc. Nat. Acad. Sci. U.S.A. vol. 28 (1942) p. 542. 


\section{Chapter IV. Continuous mappings}

19. Equivariant mappings. The following assumptions will be made throughout this chapter.

$X$ and $Y$ are topological spaces, with the group $W$ operating on both of them without fixed points. $f: X \rightarrow Y$ is a continuous mapping which is equivariant:

$$
f(w x)=w f(x) \quad w \in W, x \in X .
$$

The map $f$ defines a chain transformation $f: S(X) \rightarrow S(Y)$ as follows. For every singular simplex $T: s \rightarrow X$ the mapping $f T: s \rightarrow Y$ is a singular simplex in $Y$. This chain transformation is equivariant and therefore defines (for every discrete coefficient group $G$ with $W$ as right operators) a mapping of the exact homology sequence of $X$ into that of $Y$ (see $\$ 5$ ).

$$
\begin{aligned}
& H_{0}^{8}(X, G) \overleftarrow{\psi} \cdots \overleftarrow{\partial} H_{q}^{e}(X, G) \overleftarrow{\psi} H_{q}(X, G) \overleftarrow{\phi} H_{q}^{r}(X, G) \leftarrow H_{q+1}^{e}(X, G) \leftarrow \cdots \\
& \downarrow f_{0}^{e} \quad \downarrow f_{q}^{e} \quad \downarrow f_{q} \quad \downarrow f_{q}^{r} \quad \downarrow f_{q+1}^{e}
\end{aligned}
$$

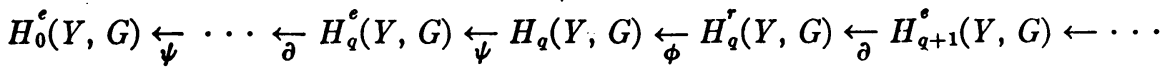

with the commutativity relations

$$
f_{q}^{\bullet} \psi=\psi f_{q}, \quad f_{q} \phi=\phi f_{q}^{r}, \quad f_{q}^{r} \partial=\partial f_{q+1}^{\bullet} .
$$

Let $\{\bar{y}\}$ be a set of representatives for $Y$. We define $\{\bar{x}\}=f^{-1}\{\bar{y}\}$. In other words $\bar{x}$ is a representative if and only if $f(\bar{x})$ is. The equivariance condition on $f$ implies that $\{\bar{x}\}$ is a set of representatives for $X$. In fact, if $\bar{x}$ and $w \bar{x}$ are both in $\{\bar{x}\}$ then $\bar{y}=f(\bar{x})$ and $f(w \bar{x})$ are both in $\{\bar{y}\}$, but $f(w \bar{x})=w f(\bar{x})=w \bar{y}$, hence $\bar{y}$ and $w \bar{y}$ are both in $\{\bar{y}\}$ and therefore $w=1$.

With the representatives $\{\bar{x}\}$ and $\{\bar{y}\}$ thus fixed we consider the chain transformations

$$
\tau_{X}: S(X) \rightarrow K_{W}, \quad \tau_{Y}: S(Y) \rightarrow K_{W}
$$

defined in $\S 14$. If $T$ is a singular $q$-simplex in $X$ with vertices $w_{0} \bar{x}_{0}, \cdots, w_{q} \bar{x}_{q}$ then the vertices of $f T$ are $f\left(w_{0} \bar{x}_{0}\right), \cdots, f\left(w_{q} \bar{x}_{q}\right)$ and since $f$ is equivariant, the vertices of $f T$ are $w_{0} f\left(\bar{x}_{0}\right), \cdots, w_{q} f\left(\bar{x}_{q}\right)$. Since $f\left(\bar{x}_{i}\right)$ are in $\{\bar{y}\}$ it follows that

$$
\tau_{Y}(f T)=\left(w_{c}, \cdots, w_{q}\right)=\tau_{X} T .
$$

Hence we obtain the fundamental relation

$$
\tau_{X}=\tau_{Y} f
$$

which implies

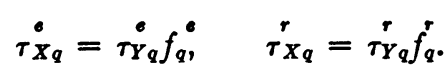

In particular it follows that the kernel of $f_{a}^{e}$ is contained in the kernel of 
$\tau_{X_{q}}^{e}$ and that the image of $\tau_{X_{q}}^{e}$ is contained in the image of $\tau_{Y_{q}}^{e}$. Similar relations hold for the residual groups.

The previous considerations, as well as the remaining results of this chapter, are also valid for cohomologies. However, in order to avoid dual formulations we shall stick to homologies throughout.

20. Principal lemma. A homomorphism $\phi: G_{1} \rightarrow G_{2}$ is called trivial if $\phi\left(G_{1}\right)$ is the zero subgroup of $G_{2}$.

LEMMA 20.1. If in addition to the previous assumptions on $W, X, Y$ and $f$ the following holds:

(20.1) $X$ is acyclic in the dimensions less than $q$ over a ring $J$ with a unit as coefficients,

(20.2) $G$ is a discrete abelian group with $W$ as right operators such that $H_{q+1}\left(W, G_{J}\right) \neq 0$,

(20.3) the homomorphism $\partial: H_{a+1}^{e}\left(Y, G_{J}\right) \rightarrow H_{a}^{r}\left(Y, G_{J}\right)$ is trivial, then the homomorphism

is not trivial.

$$
f_{q}: H_{q}\left(X, G_{J}\right) \rightarrow H_{q}\left(Y, G_{J}\right)
$$

Proof. Consider the homomorphism

$$
\tau_{X_{q}}^{r}: H_{q}^{r}\left(X, G_{J}\right) \rightarrow H_{q}^{r}\left(K_{W}, G_{J}\right) .
$$

By the results of Chapter III, $\tau_{\mathbb{X}_{q}}^{r}$ is a mapping onto, and since $H_{q}^{r}\left(K_{W}, G_{J}\right)$ $\approx H_{q+1}\left(W, G_{J}\right) \neq 0$ it follows that $\tau_{X_{q}}^{r}$ is not trivial. However $\tau_{X_{q}}^{r}=\tau_{Y q}^{r} f_{q}^{r}$ hence $f_{a}^{r}$ is not trivial. By (20.3) and the exactness property of the sequences, $\phi: H_{q}^{r}\left(Y, G_{J}\right) \rightarrow H_{q}\left(Y, G_{J}\right)$ is an isomorphism into. Hence $\phi f_{q}^{r}$ is not trivial. By the commutativity relations, $\phi f_{q}^{r}=f_{q} \phi$. Consequently $f_{q}$ is not trivial.

21. Periodic transformations. The case when $W$ is a cyclic group reduces to the case when a periodic transformation is given in $X$ and in $Y$.

THEOREM 21.1. If

(21.1) $X$ is a topological space with a periodic transformation $\Lambda: X \rightarrow X$ of prime period $p$,

(21.2) $P$ is a simplicial polyhedron of dimension not greater than $q$, with a simplicial periodic transformation $\Lambda: P \rightarrow P$ of period $p$ without fixed points, $x \in X$,

(21.3) $f: X \rightarrow P$ is a continuous mapping such that $f \Lambda(x)=\Lambda f(x)$ for each

(21.4) $X$ is acyclic in dimensions less than $q$ over some ring $J$ with a unit in. which the equation $p x=1$ has no solution,

then the homomorphisms

$$
f_{q}: H_{q}\left(X, J_{p}\right) \rightarrow H_{q}\left(P, J_{p}\right), \quad f_{q}: H_{q}(X, J) \rightarrow H_{q}(P, J)
$$

are not trivial ( $\left.{ }^{(}\right)$.

( ${ }^{\circ}$ The following notations will be used. For every integer $m,{ }_{m} J$ is the kernel of the mapping $x \rightarrow m x$ of $J$ into itself. $m J$ is the image of this homomorphism and $J_{m}=J / m J$. 
Proof. Let $W$ denote a cyclic group of order $p$ generated by an element $w_{0}$. Setting $w_{0} x=\Lambda(x)$ for $x \in X$ or $x \in P$ we find that $W$ operates on both $X$ and $P$, and that $f: X \rightarrow P$ is an equivariant map. Moreover since $\Lambda: P \rightarrow P$ has no fixed points and $p$ is prime, $W$ operates on $P$ without fixed points. Since $f$ is equivariant $W$ has no fixed points on $X$ either.

Since $w$ operates simplicially on $P$, the equivariant homology groups of $P$ can be calculated from the complex $k(P)$, hence $H_{q+1}^{e}(P, G)=0$ for any $G$ since $P$ has no $(q+1)$-simplexes. Thus condition (20.3) is fulfilled.

The homology groups $H_{q}(W, G)$ have been calculated when $W$ operates on $G$ trivially (see [6, p. 490] for the cohomology groups and [10, p. 61] for the homology groups). They are $H_{2 n}(W, G) \approx{ }_{p} G, H_{2 n+1}(W, G) \approx G_{p}$.

In particular, if $G=J_{p}$ we have $p\left(J_{p}\right)=J_{p}$ and $\left(J_{p}\right)_{p}=J_{p}$ so that $H_{n}\left(W, J_{p}\right)$ $\approx J_{p}$. The condition that the equation $p x=1$ has no solution in $J$ is equivalent with the condition that ${ }_{p} J \neq J$, or equivalently, that $J_{p} \neq 0$. Hence $H_{q+1}\left(W, J_{p}\right) \neq 0$. Since $J_{p}=I_{p} \circ J=\left(I_{p}\right)_{J}$ where $I$ is the additive group of integers, it follows that Lemma 20.1 can be applied to show that $f_{q}: H_{q}\left(X, J_{p}\right)$ $\rightarrow H_{q}\left(P, J_{p}\right)$ is not trivial. This proves the first part of the conclusion. The proof of the second half breaks up into several cases.

(A) $q$ is even. In this case $H_{q+1}(W, J) \approx J_{p} \neq 0$. Hence Lemma 20.1 can be applied to prove the desired conclusion.

(B) $q$ is odd and ${ }_{p} J \neq 0$. In this case $H_{q+1}(W, J) \approx{ }_{p} J \neq 0$ and again an application of Lemma 20.1 gives the desired result.

(C) ${ }_{p} J=0$. Consider the mapping $x \rightarrow p x$ for $x \in J$. Since the kernel of this mapping is $p J$ it follows that $p J$ is isomorphic as a group with the group of $J$. Consequently $H_{q-1}(X, p J)=0$.

Given any chain $c$ in $X$ with coefficients in $J$ we shall denote by $c_{p}$ the chain with coefficients in $J_{p}$ obtained by reducing the coefficients of $c \bmod p J$.

We already know that the homomorphism $f_{q}: H_{q}\left(X, J_{p}\right) \rightarrow H_{q}\left(P, J_{p}\right)$ is not trivial. Let then $z \in Z_{q}\left(X, J_{p}\right)$ be a cycle such that $f(Z)$ does not bound in $P$. Let $c \in C_{q}(X, J)$ be a chain such that $c_{p}=z$. Since $\partial z=0$, we have $(\partial c)_{p}=0$ and therefore $\partial c \in Z_{q-1}(X, p J)$. Since $H_{q-1}(X, p J)=0$ there is a chain $d \in C_{q}(X, p J)$ with $\partial d=\partial c$, and $d_{p}=0$. Define $z_{1}=c-d$. Since $\partial z_{1}=\partial c-\partial d=0$, $z_{1} \in Z_{q}(X, J)$.

We shall prove that $f\left(z_{1}\right)$ does not bound in $P$. Assume to the contrary that $f\left(z_{1}\right)=\partial c_{1}$ for some $c_{1} \in C_{q}(P, J)$. Then

$$
\partial\left(c_{1 p}\right)=\left(\partial c_{1}\right)_{p}=f\left(z_{1}\right)_{p}=f\left(z_{1 p}\right)=f\left(c_{p}-d_{p}\right)=f(z)
$$

contrary to assumption. This concludes the proof.

Theorem 21.1 can be used to prove a fixed point theorem for periodic transformations (see [2, p. 434]).

\section{Chapter V. Local Coefficients and COVERINGS}

22. Fundamental group, coverings. In this chapter $X$ will denote an arc- 
wise connected topological space. If $A$ is a curve from $x$ to $y$ in $X$, the class of curves from $x$ to $y$ homotopic to $A$ with end points fixed will be denoted by symbols such as $\alpha_{x y}$. The class containing the inverse path is denoted by $\alpha_{x y}^{-1}$. The symbol $\alpha_{x y} \beta_{y z}$ stands for the class obtained by traversing first a path in the class $\alpha_{x y}$ followed by a path in the class $\beta_{y z}$.

We choose a definite point $x_{0} \in X$ as base point in all future considerations. Classes of paths $\alpha_{x_{0} x}$ will be written as $\alpha_{x}$. Classes of "closed" paths $\alpha_{x_{0} x_{0}}$ will be written without any subscripts at all. For two such classes $\alpha, \beta$ the product $\alpha \beta$ is always defined, and has all the properties of a group multiplication. The resulting group is denoted by $\pi_{1}(X)$ and is called the fundamental (or Poincaré) group of $X$ with $x_{0}$ as base point.

Let $R$ be a subgroup of $\pi_{1}(X)$. We define the covering space $X_{R}$ of the space $X$ relative to the subgroup $R$ as follows. Two classes $\alpha_{x}$ and $\beta_{x}$ (both beginning at $x_{0}$ and ending at any point $x$ ) will be called equivalent $\bmod R$ if $\alpha_{x} \beta_{x}^{-1} \in R$; these equivalence classes are the points of $X_{R}$. Given a class $\alpha_{x}$ and an open set $A \subset X$ containing $x$ we define an open set $\left(\alpha_{x}, A\right)$ in $X_{R}$ by considering all the classes of the form $\alpha_{x} \beta_{x y}$ where $\beta_{x y}$ is any class containing a path in $A$. With this topology it can be shown that $X_{R}$ is an arcwise connected topological space.

The base point $x_{0 R}$ of $X_{R}$ is chosen to be the point given by the unit element of $\pi_{1}(X)$. It can be shown that $\pi_{1}\left(X_{R}\right) \approx R$.

A continuous mapping $U: X_{R} \rightarrow X$ is defined by the correspondence $\alpha_{x} \rightarrow x$. The "projection" $U$ has the following important property:

(22.1) Given any arcwise and locally arcwise connected space $Y$ such that $\pi_{1}(Y)=0$, every continuous mapping $f: Y \rightarrow X$ can be "lifted" into $X_{R}$, that is, there is a continuous mapping $\bar{f}: Y \rightarrow X_{R}$ such that $f=U \bar{f}$.

Let $\alpha$ be an element of the normalizer $N(R)$ of the subgroup $R$ (that is, $\alpha R=R \alpha)$. The correspondence $\beta_{x} \rightarrow \alpha \beta_{x}$ defines then a homeomorphism $\alpha: X_{R} \rightarrow X_{R}$ called the covering transformation corresponding to $\alpha$; it satisfies the condition $U \alpha=U$. The covering transformation $\alpha: X_{R} \rightarrow X_{R}$ is the identity map if and only if $\alpha \in R$. Hence the group $N(R) / R$ operates on $X_{R}$.

Coverings $X_{R}$ corresponding to an invariant subgroup $R$ are called regular. In this case $\pi_{1}(X)=N(R)$ and $\pi_{1}(X) / R$ operates on $X_{R}$.

If $R$ is taken to be the trivial subgroup of $\pi_{1}(X)$, the resulting covering $X_{R}=X^{\sim}$ is called the universal covering of $X$. Clearly $\pi_{1}(X)$ operates on $X^{\sim}$. The base point of $X^{\sim}$ is denoted by $x_{0}$.

23. Local groups (Steenrod [17]). We shall say that we have a system of local groups $\left\{G_{x}\right\}$ in the space $X$ if:

(23.1) For each point there is given a group $G_{x}$,

(23.2) For each class of paths $\alpha_{x y}$ an isomorphism $G_{x} \rightarrow G_{y}$ is given (we shall write $g \alpha_{x y}$ for the transform of $g \in G_{x}$ ),

(23.3) $\left(g \alpha_{x y}\right) \beta_{y z}=g\left(\alpha_{x y} \beta_{y z}\right)$.

The group $G_{x_{0}}$ where $x_{0}$ is the base point of $X$ will be denoted by $G$ with- 
out a subscript. Every element $\alpha \in \pi_{1}(X)$ gives an endomorphism $g \rightarrow g \alpha$ of $G$ so that $\pi_{1}(X)$ is a group of right operators for $G$. If $\pi_{1}(X)$ operates on $G$ simply (that is, if $g \alpha=g$ for all $g$ and $\alpha$ ) then we say that the system $\left\{G_{x}\right\}$ is simple.

Consider a singular simplex $T$ in $X$. $T$ is a mapping $T: s \rightarrow X$ where $s=\left\langle p_{0} \cdots p_{q}\right\rangle$ is a euclidean simplex with ordered vertices $p_{0}<\cdots<p_{q}$. With the singular simplex $T$ we associate the group $G_{T}=G_{T\left(p_{0}\right)}$ corresponding to its leading vertex $T\left(p_{0}\right)$. If $T^{(i)}$ denotes as before the $i$ th face of $T$ then we notice that $G_{T}=G_{T^{(i)}}$ for $i=1, \cdots, q$. For $i=0$ this may not be true since the face $T^{(0)}$ has $T\left(p_{1}\right)$ as its leading vertex. Consider then the leading edge

$$
\overline{p_{0} p_{1}} \text {; }
$$

its image

$$
\rho_{T}=T\left(\overline{p_{0} p_{1}}\right)
$$

is a path in $X$ from $T\left(p_{0}\right)$ to $T\left(p_{1}\right)$ and yields an isomorphism $g \rightarrow g_{\rho_{T}}$ of $G_{T}$ onto $G_{T(0)}$.

With these preliminaries we can now define the $q$ th homology group $H_{q}\left(X,\left\{G_{x}\right\}\right)$ of the space $X$ over the local system $\left\{G_{x}\right\}$. A chain in $X$ over $\left\{G_{x}\right\}$ is a finite formal sum

$$
\sum g_{i} T_{i},
$$

where $T_{i}$ is a singular $q$-simplex in $X$ and $g_{i} \in G_{T_{i}}$. These chains form an additive group $C_{q}\left(X,\left\{G_{x}\right\}\right)$ with addition defined by adding the coefficients within each group $G_{T_{i}}: g T+g^{\prime} T=\left(g+g^{\prime}\right) T$. The boundary of a chain $g T$ is defined as

$$
\partial(g T)=\left(g \rho_{T}\right) T^{(0)}+\sum_{i=1}^{q}(-1)^{i} g T^{(i)} .
$$

The verification that $\partial \partial=0$ is straightforward. In the usual manner we then define the groups of cycles $Z_{q}\left(X,\left\{G_{x}\right\}\right)$, boundaries $B_{q}\left(X,\left\{G_{x}\right\}\right)$, and the homology groups $H_{q}\left(X,\left\{G_{x}\right\}\right)=Z_{q}\left(X,\left\{G_{x}\right\}\right) / B_{q}\left(X,\left\{G_{x}\right\}\right)$.

In order to define cohomology groups $H^{q}\left(X,\left\{G_{x}\right\}\right)$ we shall assume that the groups $G_{x}$ are topological and that the isomorphisms $g-g \alpha_{x y}$ of $G_{x}$ into $G_{y}$ are continuous. It will further be convenient to use the inverse isomorphism $G_{y} \rightarrow G_{x}$ and write $g \rightarrow \alpha_{x y} g$ for $g \in G_{y}$. Clearly $\alpha_{x y} g=g \alpha_{x y}{ }^{-1}$. With this way of writing $\pi_{1}(X)$ becomes a group of left operators on the group $G$.

A $q$-dimensional cochain $f$ on $X$ over $\left\{G_{x}\right\}$ is defined as a function which to each singular $q$-simplex $T$ in $X$ assigns a value $f(T)$ in the group $G_{T}$. Cochains are added by adding their functional values in each group $G_{T}$. In the group of cochains $C^{q}\left(X,\left\{G_{x}\right\}\right)$ thus obtained a topology is introduced as follows. Given a singular $q$-simplex $T$ and given any open set $U$ in $G_{T}$ containing the zero of $G_{T}$ the set $(T, U)$ of all cochains $f$ with $f(T) \subset U$ is defined as a neighborhood of zero in $C^{q}\left(X,\left\{G_{x}\right\}\right)$. 
The coboundary of a cochain $f \in C^{q}\left(X,\left\{G_{x}\right\}\right)$ is defined to be the cochain $\delta f$ defined for each $(q+1)$-simplex $T$ by the formula

$$
(\delta f)(T)=\rho_{T}\left[f\left(T^{(0)}\right)\right]+\sum_{i=1}^{q+1}(-1)^{i} f\left(T^{(i)}\right) .
$$

We omit the proof that $\delta$ is strongly continuous and that $\delta \delta=0$. The groups of cocycles $Z^{q}\left(X,\left\{G_{x}\right\}\right)$ and of coboundaries $B^{q}\left(X,\left\{G_{x}\right\}\right)$ are defined in the usual way. The cohomology groups are defined as quotients $H^{q}\left(X,\left\{G_{x}\right\}\right)$ $=Z^{q}\left(X,\left\{G_{x}\right\}\right) / B^{q}\left(X,\left\{G_{x}\right\}\right)$.

It should be noted that the ordinary homology and cohomology groups $H_{q}(X, G)$ and $H^{q}(X, G)$ are included in the previous definition if we define $G_{x}=G$ for all $x \in X$ and define all the isomorphisms $G_{x} \rightarrow G_{y}$ to be identities.

24. Comparison of local and equivariant homologies. The main result of this chapter is the following theorem:

THEOREM 24.1. The homology group $H_{q}\left(X,\left\{G_{x}\right\}\right)$ of the space $X$ over the local system of groups $\left\{G_{x}\right\}$ is isomorphic with the equivariant homology group $H_{q}^{e}\left(X^{\sim}, G\right)$ of the universal covering space $X^{\sim}$ of $X$ with the fundamental group $\pi_{1}(X)$ operating on $X$ as covering transformations and operating (on the right) on the coefficient group $G=G_{x_{0}}$ by means of $g \rightarrow g \alpha$.

As a particular case we obtain the following theorem.

THEOREM 24.2. The homology group $H_{q}(X, G)$ is isomorphic with the equivariant homology group $H_{q}^{e}\left(X^{\sim}, G\right)$ of the universal covering space $X^{\sim}$ of $X$ with the fundamental group $\pi_{1}(X)$ operating on $X$ as covering transformations and operating simply on the coefficient group $G$.

Although the second theorem is a special case of the first one, its proof is much simpler and it will perhaps be useful to sketch this proof first. Consider the mapping $U: X^{\sim} \rightarrow X$. It induces homomorphisms $U: C_{q}\left(X^{\sim}, G\right) \rightarrow C_{q}(X, G)$. Since by (22.1) every singular simplex in $X$ can be "lifted" into $X^{\sim}$ these homomorphisms are "onto." It is easy to see that the group $C_{\boldsymbol{q}}^{r}\left(X^{\sim}, G\right)$ of the residual chains is the kernel of the homomorphism $U$. Since the homomorphisms $U$ commute with $\partial$ this implies that the groups $Z_{a}^{e}\left(X^{\sim}, G\right)$ and $B_{q}^{e}\left(X^{\sim}, G\right)$ are the counter-images of the groups $Z_{q}(X, G)$ and $B_{q}(X, G)$. This shows that $H_{q}^{e}\left(X^{\sim}, G\right) \approx H_{q}(X, G)$.

Proof of Theorem 24.1. Let $x \in X^{\sim}$. By definition $x$ is a homotopy class of paths in $X$ leading from the base point $x_{0}$ to the point $U x$. Consequently for each $g \in G$ the element $g x \in G_{U x}$ is well defined. Since $\alpha x$ for $\alpha \in \pi_{1}(X)$ was defined as the point of $X^{\sim}$ obtained by taking the closed path $\alpha$ first and then the path $x$ it follows from (26.3) that

$$
g(\alpha x)=(g \alpha) x .
$$

Consider a singular simplex $T$ in $X^{\sim}$; the "projection" $U T$ is then a singular 
simplex in $X$ and because of (22.1) all the singular simplexes in $X$ are of this form. Also if $U T_{1}=U T_{2}$ then $T_{2}=\alpha T_{1}$ for some $\alpha \in \pi_{1}(X)$.

Given a $q$-chain

$$
c=\sum g_{i} T_{i}, \quad g_{i} \in G,
$$

in $C_{q}\left(X^{\sim}, G\right)$, define

$$
U^{e} \mathrm{c}=\sum\left(g_{i} x_{i}\right) U T_{i},
$$

where $x_{i}$ is the leading vertex of $T_{i}$. Since $g_{i} x_{i} \in G_{T_{i}}$ it follows that $U^{e} c \in C_{q}\left(X,\left\{G_{x}\right\}\right)$. We thus get homomorphisms $U^{e}$ of the groups $C_{q}\left(X^{\sim}, G\right)$ onto the groups $C_{q}\left(X,\left\{G_{x}\right\}\right)$. In order to show that $\partial U^{e}=U^{e} \partial$ we compute

$$
U^{e}(\partial g T)=U^{e}\left(\sum_{i=0}^{q}(-1)^{i} g T^{(i)}\right)=\sum_{i=0}^{q}(-1)^{i}\left[g x^{(i)}\right] U T^{(i)},
$$

where $x^{(i)}$ is the leading vertex of $T^{(i)}$. Let $x$ be the leading vertex of $T$; then $x^{(i)}=x$ for $i>0$. The vertex $x^{(0)}$ considered as a class of paths in $X$ is the path $x$ followed by the leading edge $\rho_{T}$ of $U T$. Consequently $g x^{(0)}=g x \rho_{U T}$. Hence

$$
U^{e}(\partial g T)=\left[(g x) \rho_{U T}\right] U T^{(0)}+\sum_{i=1}^{q}(-.1)^{i}[g x] U T^{(i)}=\partial[(g x) U T]=\partial\left[U^{e}(g T)\right] .
$$

If $c \in C_{q}\left(X^{\sim}, G\right)$ is of the form

$$
c=(g \alpha) T-g(\alpha T),
$$

then $U^{\circ} c=[(g \alpha) x] U T-g(\alpha x) U \alpha T$, where $x$ is the leading vertex of $T$ and $\alpha x$ is the leading vertex of $\alpha T$. But $(g \alpha) x=g(\alpha x)$ and $U T=U \alpha T$ so that $U^{e} c=0$. Thus $C_{q}^{r}\left(X^{\sim}, G\right)$ is contained in the kernel of the homomorphism $U^{e}: C_{q}\left(X^{\sim}, G\right)-C_{q}\left(X,\left\{G_{x}\right\}\right)$. We shall now show that $C_{q}^{r}\left(X^{\sim}, G\right)$ is precisely the kernel of $U^{e}$. Let then $c \in C_{q}\left(X^{\sim}, G\right)$ and $U^{e} c=0$. Clearly we may assume that all the simplexes occurring in $c$ project upon the same singular simplex of $X$. The chain $c$ has then the form

$$
c=\sum g_{i}\left(\alpha_{i} T\right) \text {. }
$$

By definition of $U^{\circ} \mathrm{C}$ we then have

$$
U^{e} C=\sum g_{i}\left(\alpha_{i} x\right) U \alpha_{i} T=\sum g_{i}\left(\alpha_{i} x\right) U T,
$$

where $x$ is. the leading vertex of $T$. If $U^{e} c=0$ then $\sum g_{i}\left(\alpha_{i} x\right)=0$ but $g_{i}\left(\alpha_{i} x\right)$ $=\left(g_{i} \alpha_{i}\right) x$ and therefore $\sum g_{i} \alpha_{i}=0$. Consequently

$$
c=\sum\left[g_{i}\left(\alpha_{i} T\right)-\left(g_{i} \alpha_{i}\right) T\right],
$$

which proves that $c \in C_{q}^{+}\left(X^{\sim}, G\right)$.

Since $\partial\left(U^{e} c\right)=U^{e}(\partial c)$ it follows that $U c$ is a cycle if and only if $\partial c \in C_{q-1}^{\tau}\left(X^{\sim}, G\right)$, that is, if and only if $c \in Z_{q}^{e}\left(X^{\sim}, G\right)$. If $U^{e} c$ is a boundary, $U^{\circ} c=\partial d$, then there is a $d^{\prime} \in C_{q+1}\left(X^{\sim}, G\right)$ such that $U^{e} d^{\prime}=d$. We then have 
$U^{e}\left(c-\partial d^{\prime}\right)=0$ and $c-\partial d^{\prime} \in C_{q}^{r}\left(X^{\sim}, G\right)$. Since $c=\left(c-\partial d^{\prime}\right)+\partial d^{\prime}$ it follows that $c \in C_{q}^{r}\left(X^{\sim}, G\right)+B_{q}\left(X^{\sim}, G\right)=B_{q}^{e}\left(X^{\sim}, G\right)$. Conversely if $c \in B_{q}^{e}\left(X^{\sim}, G\right)$ then $c=c_{1}+\partial d$ with $c_{1} \in C_{q}^{\gamma}(X, G)$ and $U^{e} c=U^{e} c_{1}+U^{e} \partial d=\partial U^{e} d$ so that $U^{e} c$ $\in B_{q}\left(X,\left\{G_{x}\right\}\right)$. We have thus shown that the groups $Z_{q}^{e}\left(X^{\sim}, G\right)$ and $B_{a}^{e}\left(X^{\sim}, G\right)$ are the counter-images under $U^{e}$ of the groups $Z_{q}\left(X,\left\{G_{x}\right\}\right)$ and $B_{q}\left(X,\left\{G_{x}\right\}\right)$. This establishes the isomorphism $H_{q}^{e}\left(X^{\sim}, G\right) \approx H_{q}\left(X,\left\{G_{x}\right\}\right)$.

25. Comparison of local and equivariant cohomologies. For cohomologies we have a theorem analogous to Theorem 24.1.

THEOREM 25.1. The cohomology group $H^{q}\left(X,\left\{G_{x}\right\}\right)$ of the space $X$ over the local system of groups $\left\{G_{x}\right\}$ is isomorphic with the equivariant cohomology group $H_{e}^{q}\left(X^{\sim}, G\right)$ of the universal covering space $X^{\sim}$ of $X$ with the fundamental group operating on $X^{\sim}$ as covering transformations and operating (on the left) on the coefficient group $G=G_{x_{0}}$ by means of $g \rightarrow \alpha g$.

As a corollary we have:

THEOREM 25.2. The cohomology group $H^{q}(X, G)$ is isomorphic with the equivariant cohomology group $H_{\bullet}^{q}\left(X^{\sim}, G\right)$ of the uniervsal covering space $X^{\sim}$ of $X$ with the fundamental group $\pi_{1}(X)$ operating on $X^{\sim}$ as covering transformations and operating on $G$ simply.

Proof of Theorem 25.1. Let $x \in X^{\sim}$, since $x$ is a homotopy class of paths in $X$ joining $x_{0}$ with $U x$, for every $g \in G_{U x}$ the element $x g \in G=G_{x_{0}}$ is well defined. As before we have

$$
x(\alpha g)=(x \alpha) g .
$$

Given any cochain $f \in C^{q}\left(X,\left\{G_{x}\right\}\right)$ we define a cochain $U_{a} f \in C^{q}\left(X^{\sim}, G\right)$ by setting for every $q$-simplex in $X$

$$
U_{\bullet} f(T)=x f(U T),
$$

where $x$ is the leading vertex of $T$. Since $f(U T) \in G_{U T}=G_{U x}$ the element $x f(U T)$ is in $G$, and $U_{e} f$ is a cochain. For every $(q+1)$-simplex in $X$ we have

$$
\delta\left[U_{e} f\right](T)=\sum_{i=1}^{q+1}(-1)^{i} U_{e} f\left(T^{(i)}\right)=\sum_{i=1}^{q+1}(-1)^{i} x^{(i)} f\left(U T^{(i)}\right),
$$

where $x^{(i)}$ is the leading vertex of the simplex $U\left(T^{(i)}\right)=(U T)^{(i)}$. For $i=1, \cdots, q+1$ we have $x^{(i)}=x$. For $i=0$ we have $x^{(0)} f\left(U T^{(0)}\right)=x \rho_{U T} f\left(U T^{(0)}\right)$ where $x$ is the leading vertex of $T$ and $\rho_{U T}$ is the path corresponding to the leading edge of $U T$. Consequently

$$
\delta\left[U_{\ell} f\right](T)=x \rho_{U T} f\left(U T^{(0)}\right)+\sum_{i=1}^{q+1}(-1)^{i} x f\left(U T^{(i)}\right)=x(\delta f)(U T)=[U \delta \delta f](T)
$$

and $\delta U_{s}=U_{e} \delta$.

If $f \neq 0$ then $f(T) \neq 0$ for some $q$-simplex $T$ in $X$. There is then a simplex 
$T^{\sim}$ in $X^{\sim}$ such that $U T^{\sim}=T$ and $U_{e} f\left(T^{\sim}\right)=x f(T) \neq 0(x$ here is the leading vertex of $T^{\sim}$ ). Consequently $U_{\&} \neq \neq 0$.

If $x$ is the leading vertex of $T$ in $X^{\sim}$ then $\alpha x$ is the leading vertex of $\alpha T$ and

$$
U_{e} f(\alpha T)=\alpha x f(U \alpha T)=\alpha[x f(U T)]=\alpha U_{e} f(T) .
$$

Consequently the cochain $U_{e} f$ is equivariant. Conversely let $h$ be any equivariant $q$-cochain in $X$ over $G$. Given any $q$-simplex $T$ in $X$ choose a $q$-simplex $T^{\sim}$ in $X^{\sim}$ covering $T$ (that is, such that $U T^{\sim}=T$ ). Consider the element $x^{-1} h\left(T^{\sim}\right)$ of $G_{T}$ where $x$ is the leading vertex of $T^{\sim}$. If $T^{\sim}$ is replaced by $\alpha T^{\sim}$ then $(\alpha x)^{-1} h\left(\alpha T^{\sim}\right)=x^{-1} \alpha^{-1} \alpha h\left(T^{\sim}\right)=x^{-1} h\left(T^{\sim}\right)$ because $h$ is equivariant. Hence the element $x^{-1} h\left(T^{\sim}\right)$ of $G_{T}$ is independent of the choice of $T^{\sim}$ and we may define a cochain $f \in C^{q}\left(X,\left\{G_{x}\right\}\right)$ by setting $f(T)=x^{-1} h\left(T^{\sim}\right)$. Since $\left(U_{e} f\right)\left(T^{\sim}\right)=x f\left(U T^{\sim}\right)=x f(T)=x x^{-1} h(T)=h(T)$ we have $U_{e} f=h$.

We have thus shown that $U$ is an isomorphic mapping of the group of cochains $C^{q}\left(X,\left\{G_{x}\right\}\right)$ onto the group of equivariant cochains $C^{q}\left(X^{\sim}, G\right)$. Since in addition $\delta U_{e}=U_{e} \delta$ it follows that $Z^{q}\left(X,\left\{G_{x}\right\}\right)$ and $B^{q}\left(X,\left\{G_{x}\right\}\right)$ are mapped isomorphically onto the groups $Z_{e}^{q}\left(X^{\sim}, G\right)$ and $B_{e}^{q}\left(X^{\sim}, G\right)$. This proves the isomorphism $H^{q}\left(X,\left\{G_{x}\right\}\right) \approx H_{c}^{q}\left(X^{\sim}, G\right)$.

26. Regular coverings. The results of the previous two sections are capable of the following generalization. Let $\left\{G_{x}\right\}$ be a local system of groups in the space $X$ and let $G=G_{x_{0}}$ where $x_{0}$ is the base point of $X$. Suppose that an invariant subgroup $R$ of $\pi_{1}(X)$ operates simply on $G$, that is, that $g \alpha=g$ for $\alpha \in R$ and all $g \in G$. The group $W=\pi_{1}(X) / R$ can then be defined as a group of operators (left for cohomologies and right for homologies) on $G$. Construct the covering $X_{R}$ of $X$ corresponding to the subgroup $R$. Since $R$ is invariant the covering is regular and $W$ operates on $X$ as a group of covering transformations. Thus $W$ operates on $X_{R}$ and $G$ and we may define the equivariant homology and cohomology groups of $X_{R}$. The previous proofs generalize to give isomorphisms

$$
H_{q}\left(X,\left\{G_{x}\right\}\right) \approx H_{q}^{e}\left(X_{R}, G\right) ; \quad H^{q}\left(X,\left\{G_{x}\right\}\right) \approx H_{e}^{q}\left(X_{R}, G\right) .
$$

27. Spherical cycles. An integral cycle $z \in Z_{q}(X)$ is called spherical if there is a continuous mapping $f: S^{q} \rightarrow X$ of the $q$-dimensional sphere $S^{q}$ into $X$ and a cycle $z^{\prime} \in Z_{q}\left(S^{q}\right)$ such that $f\left(z^{\prime}\right)$ is homologous to $z$ in $X$. It is well known that in an arcwise connected space $X$, the spherical cycles form a group containing the group $B_{q}(X)$. Hence the spherical cycles determine a subgroup $\Sigma_{q}(X)$ of the homology group $H_{q}(X)$. The group $\Sigma_{q}(X)$ can also be defined as the image of the $q$ th homotopy group $\pi_{q}(X)$ of $X$ under the natural homomorphism $\pi_{q}(X) \rightarrow H_{q}(X)$.

A cycle $z \in Z_{q}(X, G)$ with coefficients in a group $G$ is called spherical provided $z=\sum_{i=1}^{n} g_{i} z^{i}$ where $g_{i} \in G$ and $z^{i}$ are spherical cycles with integer coefficients. We thus obtain a subgroup $\Sigma_{q}(X, G)$ of $H_{q}(X, G)$.

Let $X^{\sim}$ be the universal covering of $X$. We can then consider the equi- 
variant homology group $H_{q}^{e}\left(X^{\sim}, G\right)$ with $\pi_{1}(X)$ operating on $X$ as covering transformations and operating on $G$ simply. We also have the homomorphism

$$
\psi: H_{q}\left(X^{\sim}, G\right) \rightarrow H_{q}^{\bullet}\left(X^{\sim}, G\right)
$$

and the isomorphism

$$
U^{e}: H_{q}^{e}\left(X^{\sim}, G\right) \approx H_{q}(X, G) .
$$

We shall show that

$$
\Sigma_{q}(X, G)=U^{e} \psi \Sigma_{q}\left(X^{\sim}, G\right) \quad \text { for } q>1 .
$$

Clearly if $z \in \Sigma_{q}\left(X^{\sim}, G\right)$ then $U \psi z \in \Sigma_{q}(X, G)$. The opposite inclusion follows from (22.1).

Let now $\left\{G_{x}\right\}$ be a local system of groups in $X$. Consider the equivariant group $H_{e}^{e}\left(X^{\sim}, G\right)$ where $\pi_{1}(X)$ operates on $X$ as covering transformations and operates on $G=G_{x_{0}}$ on the right by means of $g \rightarrow g \alpha$. We have again the homomorphism (27.1) and the isomorphism

$$
U^{e}: H_{q}^{e}(X, G) \approx H_{q}\left(X,\left\{G_{x}\right\}\right) .
$$

We now define the spherical subgroup of $H_{q}\left(X,\left\{G_{x}\right\}\right)$ as

$$
\Sigma_{q}\left(X,\left\{G_{x}\right\}\right)=U \cdot \psi \Sigma_{q}(X, G),
$$

Formula (27.2) shows that this definition is compatible with the definition of $\Sigma_{q}(X, G)$ given earlier in this section.

Passing to cohomology, we say that a cocycle $f \in Z^{q}(X, G)$ is a spherical annihilator if $f(z)=0$ for every integral spherical cycle $z \in Z_{q}(X)$. The spherical annihilators determine a subgroup $\Lambda^{q}(X, G)$ of $H^{q}(X, G)$.

Let now $\left\{G_{x}\right\}$ be a local system of (topological) groups in $X$. Consider the equivariant cohomology group $H_{e}^{q}\left(X^{\sim}, G\right)$ where $\pi_{1}(X)$ operates on $X^{\sim}$ as covering transformations and operates on $G=G_{x_{0}}$ on the left by means of $g \rightarrow \alpha g$. We have the homomorphism

$$
\phi: H_{e}^{q}\left(X^{\sim}, G\right) \rightarrow H^{q}\left(X^{\sim}, G\right)
$$

and the isomorphism

$$
U_{e}: H^{q}\left(X,\left\{G_{x}\right\}\right) \approx H_{e}^{q}\left(X^{\sim}, G\right) .
$$

We define the subgroup $\Lambda^{q}\left(X,\left\{G_{x}\right\}\right)$ of $H^{q}\left(X,\left\{G_{x}\right\}\right)$ as

$$
\Lambda^{q}\left(X,\left\{G_{x}\right\}\right)=\left(\phi U_{e}\right)^{-1} \Lambda^{q}\left(X^{\sim}, G\right), \quad q>1 .
$$

As before we show that the definition in the case of local groups is compatible with the definition of $\Lambda^{q}(X, G)$ given above.

As a preliminary to the next section we prove the following lemma.

Lemma 27.1. If $X$ is arcwise connected and if $\pi_{i}(X)=0$ for $i<q$ then $X$ is 
acyclic in dimensions less than $q$ and

$$
\Sigma_{q}(X, G)=H_{q}(X, G), \quad \Lambda^{q}(X, G)=0 .
$$

Proof. The fact that $H_{i}(X)=0$ for $0<i<q$ and that $\pi_{q}(X) \approx \Sigma_{q}(X)$ $=H_{q}(X)$ is well known $\left[12_{2}\right]$.

Let $z \in Z_{q}(X, G)$. Since $G$ is an abelian discrete group it is the image $\phi: F \rightarrow G$ of a free abelian group $F$ under a homomorphism $\phi$ with kernel $R$. The homomorphism $\phi$ induces a homomorphism $\phi: C_{q}(X, F) \rightarrow C_{q}(X, G)$ which maps $C_{q}(X, F)$ onto $C_{q}(X, G)$. Let $c \in C_{q}(X, F)$ be such that $\phi(c)=z$. Since $\partial z=0$ it follows that $\partial c \in Z_{q-1}(X, R)$. As a subgroup of a free group $F, R$ itself is free, and since $H_{q-1}(X)=0$ it follows that $H_{q-1}(X, R)=0$. Hence there is a $d \in C_{q}(X, R)$ such that $\partial d=\partial c$. Let $z^{\prime}=c-d$, then $z^{\prime} \in Z_{q}(X, F)$ and $\phi\left(z^{\prime}\right)=z$. Since $z^{\prime}$ can be written as $\sum a_{i} z_{i}$ with $a_{i} \in F$ and $z_{i} \in Z_{q}(X)$ it follows that $z=\Sigma \phi\left(a_{i}\right) z_{i}$. Since all the $z_{i}$ are spherical, it follows that $z$ is spherical and $\Sigma_{q}(X, G)=H_{q}(X, G)$.

In order to show that $\Lambda^{q}(X, G)=0$ consider a spherical annihilator $f^{a} \in Z^{q}(X, G)$. Since all cycles in $Z_{q}(X)$ are spherical it follows that the homomorphism $f^{q}: C_{q}(X) \rightarrow G$ maps $Z_{q}(X)$ into 0 . Since $Z_{q-1}(X)=B_{q-1}(X)$ $=C_{q}(X) / Z_{q}(X)$ it follows that $f^{q}$ defines a homomorphism $g^{q-1}: Z_{q-1}(X) \rightarrow G$ such that $g^{q-1}(\partial c)=f^{q}(c)$ for $c \in C_{q}(X)$. Since $Z_{q-1}(X)$ is a direct summand of $C_{q-1}(X)$, the homomorphism $g^{q-1}$ can be extended to a homomorphism $h^{q-1}: C_{q-1}(X) \rightarrow G$. Since $\partial h^{q-1}(c)=h^{q-1}(\partial c)=g^{q-1}(\partial c)=f^{q}(c)$ it follows that $f^{q}=\delta h^{q-1}$ and $\Lambda^{q}(X, G)=0$.

28. Relations between fundamental group and homology groups. Combining the results of this chapter with those of chapter III we arrive at a series of formulae linking the fundamental group $\pi_{1}(x)$ with the homology and cohomology groups of $X$.

THEOREM 28.1. Let $X$ be an arcwise connected topological space such that (with base point $x_{0}$ )

$$
\pi_{i}(X)=0
$$$$
\text { for } 1<i<q \text {. }
$$

For every local system $\left\{G_{x}\right\}$ of discrete abelian groups on $X$, the following isomorphisms hold:

$$
\begin{aligned}
H_{i}\left(X,\left\{G_{x}\right\}\right) & \approx H_{i}\left(\pi_{1}(X), G\right), \quad i=1, \cdots, q-1, \\
H_{q}\left(X,\left\{G_{x}\right\}\right) / \Sigma_{q}\left(X,\left\{G_{x}\right\}\right) & \approx H_{q}\left(\pi_{1}(X), G\right),
\end{aligned}
$$

where $G=G_{x_{0}}$ and $\pi_{1}(X)$ operates on $G$ (on the right) by means of $g \rightarrow g \alpha$.

For every local system $\left\{G_{x}\right\}$ of topological abelian groups on $X$, the following isomorphisms hold:

$$
\begin{aligned}
H^{i}\left(X,\left\{G_{x}\right\}\right) & \approx H^{i}\left(\pi_{1}(X), G\right), \quad i=1, \cdots, q-1, \\
\Lambda^{q}\left(X,\left\{G_{x}\right\}\right) & \approx H^{q}\left(\pi_{1}(X), G\right),
\end{aligned}
$$

where $G=G_{x_{0}}$ and $\pi_{1}(X)$ operates on $G$ (on the left) by means of $g \rightarrow \alpha g$. 
Proof. Let $X^{\sim}$ be the universal covering space of $X$ with $\pi_{1}(X)$ operating on $X$ as covering transformations. Since $\pi_{i}\left(X^{\sim}\right) \approx \pi_{i}(X)$ for $i>1$ (this is an easy consequence of $(22.1))$, it follows that $\pi_{i}\left(X^{\sim}\right)=0$ for $1<i<q$. Since in addition $\pi_{i}\left(X^{\sim}\right)=0$ and $X^{\sim}$ is arcwise connected it follows from Lemma 27.1 that $X^{\sim}$ is acyclic in dimensions less than $q$. Hence Theorem 16.1 can be applied. For $i<q$ we have the isomorphisms

$$
H_{i}^{e}\left(X^{\sim}, G\right) \approx H_{i}\left(\pi_{1}(X), G\right), \quad H_{e}^{i}\left(X^{\sim}, G\right) \approx H^{i}\left(\pi_{1}(X), G\right),
$$

while Theorem 24.1 and 25.1 give isomorphisms

$$
H_{i}\left(X,\left\{G_{x}\right\}\right) \approx H_{i}^{e}\left(X^{\sim}, G\right), \quad H^{i}\left(X,\left\{G_{x}\right\}\right) \approx H_{e}^{i}\left(X^{\sim}, G\right) .
$$

Combining these isomorphisms leads to (28.1) and (28.3).

In the dimension $q$, Theorem 16.1 asserts that

$$
H_{q}^{e}\left(X^{\sim}, G\right) / \psi H_{q}(X, G) \approx H_{q}\left(\pi_{1}(X), G\right) .
$$

Under the isomorphism $U^{e}$ the left-hand side is transformed isomorphically onto $H_{q}\left(X,\left\{G_{x}\right\}\right) / U^{e} \psi H_{q}\left(X^{\sim}, G\right)$. Since, by Lemma 27.1, $H_{q}\left(X^{\sim}, G\right)$ $=\Sigma_{q}\left(X^{\sim}, G\right)$, we have $U^{e} \psi H_{q}(X, G)=U^{e} \psi \Sigma_{q}(X, G)=\Sigma_{q}\left(X,\left\{G_{x}\right\}\right)$. Hence the left-hand side of (28.5) is isomorphic with $H_{q}\left(X,\left\{G_{x}\right\}\right) / \Sigma_{q}\left(X,\left\{G_{x}\right\}\right)$, thus proving (28.2).

For cohomology, Theorem 16.1 asserts that

$$
K^{q}\left(X^{\sim}, G\right) \approx H^{q}\left(\pi_{1}(X), G\right),
$$

where $K^{q}\left(X^{\sim}, G\right)$ is the kernel of the homomorphism $\phi: H_{e}^{q}\left(X^{\sim}, G\right)$ $\rightarrow H^{q}\left(X^{\sim}, G\right)$. By Lemma $27.1, \Lambda^{q}\left(X^{\sim}, G\right)=0$ so that $K^{q}\left(X^{\sim}, G\right)$ $=\phi^{-1} \Lambda^{q}\left(X^{\sim}, G\right)$. Under the inverse of the isomorphism $U_{e}$ the group $K^{q}(X, G)$ is transformed isomorphically onto the group $U_{e}^{-1} \phi^{-1} \Lambda^{q}\left(X^{\sim}, G\right)$ $=\left(\phi U_{\theta}\right)^{-1} \Lambda^{q}\left(X^{\sim}, G\right)=\Lambda^{q}\left(X,\left\{G_{x}\right\}\right)$. Hence $\Lambda^{q}\left(X,\left\{G_{x}\right\}\right) \approx K^{q}\left(X^{\sim}, G\right)$ under the isomorphism $U_{e}$. This combined with (28.6) yields (28.4).

\section{Appendix. Products}

We shall briefly show how the cup product of cochains is related to the equivariant theory developed in the body of the paper.

If $G_{1}$ and $G_{2}$ are two topological abelian groups paired [3, p. 415] to a third such group $G$ and if the group $W$ operates on the left on $G_{1}, G_{2}$ and $G$ then in addition to the usual properties of the pairing we shall also assume that

$$
w\left(g_{1} \cup g_{2}\right)=w g_{1} \cup w g_{2},
$$

$$
g_{i} \in G_{1}, g_{2} \in G_{2}, w \in W .
$$

In $[3, \S 28]$ we have introduced cup products of cochains in the complexes $S(X)$ and $K(P)$. If now $W$ operates on the space $X$ (or operates simplicially on $P$ ) then it can be verified immediately that the cup product $f_{1} \cup f_{2}$ of two equivariant cochains is again equivariant. Thus the equivariant cohomology 
groups $H_{e}^{m}\left(X, G_{1}\right)$ and $H_{e}^{n}\left(X, G_{2}\right)$ are paired to the group $H_{e}^{m+n}(X, G)$. The same holds in the complex $K(P)$. Since the chain transformation $\beta: K(P)$ $\rightarrow S(P)$ defined in $\S 9$ preserves the product $[3, \mathrm{p} .435]$ it follows that the isomorphisms between the various groups of $K(P)$ and $S(P)$ asserted in Corollary 10.2 preserve the product.

Next we define products in the complex $K_{W}$ of the group $W$. Given two cochains $f_{1}^{m} \in C^{m}\left(K_{W}, G_{1}\right)$ and $f_{2}^{n} \in C^{n}\left(K_{W}, G_{2}\right)$ we define the cochain $f_{1}^{m} \cup f_{2}^{n}$ $\in C^{m+n}\left(K_{W}, G\right)$ by setting

$$
f_{1}^{m} \cup f_{2}^{n}\left(w_{0}, \cdots, w_{m+n}\right)=f_{1}^{m}\left(w_{0}, \cdots, w_{m}\right) \cup f_{2}^{n}\left(w_{m}, \cdots, w_{m+n}\right) .
$$

This defines a cup product in $K_{W}$. Again it follows from condition (A.1) that the cup product of two equivariant cochains is equivariant and therefore that the groups $H^{m}\left(W, G_{1}\right)$ and $H^{n}\left(W, G_{2}\right)$ are paired to the group $H^{m+n}(W, G)$.

A glance at the definition (\$14) of the chain transformation $\tau: S(X) \rightarrow K_{W}$ shows that $\tau$ preserves the products. It follows that for $m+n<q$ the isomorphisms

$$
\begin{gathered}
H_{e}^{m}\left(X, G_{1}\right) \approx H^{m}\left(W, G_{1}\right), \quad H_{e}^{n}\left(X, G_{2}\right) \approx H^{n}\left(W, G_{2}\right), \\
H_{e}^{m+n}(X, G) \approx H^{m+n}(W, G)
\end{gathered}
$$

preserve the products.

If $m+n=q$ and $m>0, n>0$, the last isomorphism is replaced by

$$
K^{m+n}(X, G) \approx H^{m+n}(W, G)
$$

where $K^{m+n}(X, G)$ is the kernel of the homomorphism $H_{a}^{m+n}(X, G)$ $\rightarrow H^{m+n}(X, G)$. A slight argument is then needed. If $f_{1}^{m}$ and $f_{2}^{n}$ are equivariant cocycles, then since $m<q, n<q$, and the space $X$ was assumed to be acyclic in dimensions less than $q$, it follows that $f_{1}^{m} \cup f_{2}^{n}$ is a coboundary (not necessarily of an equivariant cochain). Hence the cohomology class of $f_{1}^{m} \cup f_{2}^{n}$ is in the subgroup $K^{m+n}(X, G)$. Therefore, if we regard $H_{b}^{m}\left(X, G_{1}\right)$ and $H_{e}^{n}\left(X, G_{2}\right)$ as paired to the group $K^{m+n}(X, G)$, then this "reduced" pairing is isomorphic with the pairing of the groups $H^{m}\left(W, G_{1}\right), H^{n}\left(W, G_{1}\right)$ to the group $H^{m+n}(W, G)$.

Cup products can also be defined for cohomology groups with local coefficients (see [17]). Two local systems $\left\{G_{x}^{1}\right\},\left\{G_{x}^{2}\right\}$ of topological abelian groups on a space $X$ will be said to be paired to a third such system $\left\{G_{x}\right\}$ provided that for each $x \in X$ the groups $G_{x}^{1}$ and $G_{x}^{2}$ are paired to $G_{x}$ in such a fashion that

$$
\left(g_{1} \cup g_{2}\right) \alpha_{x y}=g_{1} \alpha_{x y} \cup g_{2} \alpha_{x y} .
$$

The cup product $f_{1}^{m} \cup f_{2}^{n}$ of two cochains $f_{1}^{m} \in C^{m}\left(X,\left\{G_{x}^{1}\right\}\right)$ and $f_{2}^{n} \in C^{n}\left(X,\left\{G_{x}^{2}\right\}\right)$ is then defined as follows. Let $T: s \rightarrow X$ be a singular simplex of dimension $m+n$ where $s$ is a euclidean simplex with ordered vertices $p_{0}, \cdots, p_{m+n}$. Let ${ }_{m} T$ be the $m$-dimensional singular simplex obtained by restricting $T$ to the ordered subsimplex of $s$ given by the vertices $p_{0}, \cdots, p_{m}$. Similarly de- 
fine $T_{n}$ by using the vertices $p_{m}, \cdots, p_{m+n}$. Further let $\alpha$ denote the path in $X$ which is the $T$-image of the edge $p_{0} p_{m}$ in $s$. Define

$$
\left(f_{1}^{m} \cup f_{2}^{n}\right)(T)=f_{1}^{m}\left({ }_{m} T\right) \cup \alpha f_{2}^{n}\left(T_{n}\right) .
$$

It is a matter of straightforward verification that the usual properties of the cup product hold and that the groups $H^{m}\left(X,\left\{G_{x}^{1}\right\}\right)$ and $H^{n}\left(X,\left\{G_{x}^{2}\right\}\right)$ are paired to the group $H^{m+n}\left(X,\left\{G_{x}\right\}\right)$. It is also easy to see that the isomorphism $U_{e}$ constructed to prove Theorem 28.1 preserves the products. The same applies to the isomorphisms in Theorem 25.1 since they are isomorphisms given by the chain transformation $\tau$.

\section{BIBLIOGRAPHY}

1. B. Eckmann, Der Cohomologie-Ring einer beliebigen Gruppe, Comment. Math. Helv. vol. 18 (1945-1946) pp. 232-282.

2. S. Eilenberg, On a theorem of $P$. A. Smith concerning fixed points for periodic transformations, Duke Math. J. vol. 6 (1940) pp. 428-437.

3. - Singular homology theory, Ann. of Math. vol. 45 (1944) pp. 407-447.

4. - Singular homology in differentiable manifolds, Ann. of Math. vol. 48 (1947).

5. S. Eilenberg and S. MacLane, Relations between homology and homotopy groups, Proc. Nat. Acad. Sci. U.S.A. vol. 29 (1943) pp. 155-158.

6. - Relations between homology and homotopy groups of spaces, Ann. of Math. vol. 46 (1945) pp. $480-509$.

7. - Cohomology theory in abstract groups I, Ann. of Math. vol. 48 (1947) pp. 51-78.

8. H. Freudenthal, Der Einfluss der Fundamentalgruppe auf die Bettischen Gruppen, Ann. of Math. vol. 47 (1946) pp. 274-316.

9,2. H. Hopf, Fundamentalegruppe und zweite Bettische Gruppe, Comment. Math. Helv. vol. 14 (1942) pp. 257-309. Nachtrag zu der Arbeit . . . ibid. vol. 15 (1943) pp. 27-32.

10. - Ueber die Bettischen Gruppen die zu einer beliebigen Gruppe gehören, Comment. Math. Helv. vol. 17 (1945) pp. 39-79. 333.

11. — , Beiträge zur Homotopietheorie, Comment. Math. Helv. vol. 17 (1945) pp. 307-

$12_{1,2,8,4 .}$ W. Hurewicz, Beilräge zur Topologie der Deformationen, Proceedings of the section of science, K. Akademie van wetenschappen, Amsterdam vol. 38 (1935) pp. 112-119, 521-528; vol. 39 (1935) pp. 117-125, 215-224.

13. J. L. Kelley and E. Pitcher, Exact homomorphism sequences and homology theory, Ann. of Math. vol. 48 (1947).

14. S. Lefschetz, Algebraic topology, Amer. Math. Soc. Colloquium Publications, vol. 27, New York, 1942.

15. G. de Rham, Sur les complexes avec automorphismes, Comment. Math. Helv. vol. 12 (1939) pp. 191-211.

16. P. A. Smith, Fixed points of periodic transformations, Appendix to [14], pp. 350-373.

17. N. E. Steenrod, Homology with local coefficients, Ann. of Math. vol. 44 (1943) pp. 610627.

UNIVERSITY OF MICHIGAN, ANN ARBOR, Mich. 